\title{
Tachyons and the Wheeler-Feynman absorber theory
}

\author{
Roman Tomaschitz \\ Department of Physics, Hiroshima University, 1-3-1 Kagami-yama, Higashi-Hiroshima \\ 739-8526, Japan \\ E-mail: roman@fusion.sci.hiroshima-u.ac.jp
}

Received 6 March 2001

Published 22 October 2001

Online at stacks.iop.org/CQG/18/4395

\begin{abstract}
The Proca equation with negative mass-square is studied in a refractive and absorptive spacetime. The generation of superluminal radiation fields by subluminal currents is discussed. The possibility of time-symmetric wave propagation is analysed in the context of the Wheeler-Feynman absorber theory; it is shown how advanced modes of the Proca field can be turned into retarded ones in a permeable spacetime capable of producing an absorber field. A microscopic oscillator model for the permeability is suggested. Tachyonic Liénard-Wiechert potentials are studied and strictly causal retarded wave solutions are obtained. Energy transfer by superluminal radiation is discussed, and explicit formulae for the spectral energy density and intensity are derived. Superluminal radiation fields generated by classical damped oscillators carrying tachyonic charge are investigated, including the tachyonic analogue to Thomson and Rayleigh cross sections. The Maxwell equations for negative mass-square are derived, their non-local generalization to frequency-dependent permeabilities, as well as the Poynting theorem for superluminal radiation in an absorptive spacetime.
\end{abstract}

PACS numbers: 9880, 0350K, 1110L, 4225B

\section{Introduction}

The traditional way of introducing superluminal particles (tachyons) is to start with the Lagrangian

$$
L=-m_{\mathrm{t}} c^{2} \sqrt{\eta_{\alpha \beta} \dot{x}^{\alpha} \dot{x}^{\beta}}+e A_{\alpha} \dot{x}^{\alpha}
$$

where $\eta_{\alpha \beta}=\operatorname{diag}\left(-c^{2}, 1,1,1\right), m_{\mathrm{t}}>0$, which differs from the Lagrangian of a classical subluminal particle just by a minus sign under the root [1-12]. The superluminal particle is coupled by minimal substitution to the electromagnetic field as indicated, if it carries electric charge $e$. In this paper a very different approach to superluminal signals is investigated, a 
Proca equation with negative mass-square, in contrast to the prevailing view of tachyons as electrically charged point particles (1.1). The superluminal Proca field is coupled to a current of subluminal massive particles [13,14]. The Lagrangians for the tachyon field and a subluminal classical particle coupled to this field read

$$
\begin{aligned}
& L_{\text {Proca }}=-\frac{1}{4} F_{\alpha \beta} F^{\alpha \beta}+\frac{1}{2}\left(m_{\mathrm{t}} c / \hbar\right)^{2} A_{\alpha} A^{\alpha}+c^{-1} A_{\alpha} j^{\alpha}, \\
& L=-m c^{2} \sqrt{-\eta_{\alpha \beta} \dot{x}^{\alpha} \dot{x}^{\beta}}+q A_{\alpha} \dot{x}^{\alpha},
\end{aligned}
$$

respectively, where $m_{\mathrm{t}}>0$ is the mass of the tachyon field $A_{\alpha}$. (The mass term in (1.2) is added with a positive sign, so that $m_{\mathrm{t}}^{2}>0$ is the negative mass-square of the tachyon field.) The subluminal particle of mass $m$ as defined by (1.3) is supposed to carry tachyonic charge $q$, by which it couples to the tachyon potential via a current $j^{0}=\rho=q \delta(\boldsymbol{x}-\boldsymbol{x}(t))$, $\boldsymbol{j}=q \boldsymbol{v} \delta(\boldsymbol{x}-\boldsymbol{x}(t))$. Evidently, the Proca Lagrangian is designed in analogy to electrodynamics, but is otherwise unrelated to electromagnetic fields, and the real vector potential $A_{\alpha}$ is itself a measurable quantity, as the mass term breaks the gauge invariance. In this way tachyons emerge as an extension of the photon concept, a sort of photon with negative mass-square (see the review [15] on electrodynamics with a photon rest mass). The tachyon field does not carry any kind of charge, the tachyonic charge $q$ is a property of subluminal particles (contrary to (1.1)), as is electric charge. In the geometrical optics limit of this field theory, one can describe superluminal rays by the Lagrangian (1.1) with the interaction term dropped.

The theory of superluminal motion presented here is causal and non-relativistic. Cosmic space is generated by the galaxy grid, which provides a distinguished reference frame manifested locally by the Planckian microwave background. The state of absolute rest can be defined with respect to the galaxy grid, and uniform motion and rest become easily distinguishable states. Whether an observer is at rest or in uniform motion with respect to the microwave background, this can unambiguously be decided by measuring the dipole anisotropy of the background temperature caused by a Doppler shift. If tachyons are defined with respect to this universal rest frame, a causality problem does not arise, since the cosmic time order of events is unambiguously defined by the comoving galaxy grid. All uniformly moving observers, irrespective of their location in the universe, can relate their proper time to cosmic time, by determining their motion relative to the background radiation, and arrive in this way at the same conclusion on causal connections. To figure out the causality of an experiment involving tachyons, one has to connect the laboratory to the rest of the universe and to determine its motion relative to the galaxy background. The solar barycentre is moving with some $370 \mathrm{~km} \mathrm{~s}^{-1}$ relative to the microwave radiation, fast enough to even neglect the relative motions of the Earth in a first approximation [16]. However, the background radiation is just a practical tool to determine the observer's velocity in the galaxy grid, a photon gas pervading space. If there is an absolute cosmic space as defined by the galaxy grid and the microwave radiation, we are again permitted to contemplate the substance of space itself, i.e. the ether [17-21]. The galaxy grid is anchored in the ether and wave propagation, classical or quantum, takes place in this permeable spacetime, the vacuum is just a geometric idealization.

There are two, essentially non-overlapping methods of introducing permeability into the Maxwell and Proca equations. Firstly, by means of a permeability tensor, $g_{\mu \nu}^{P}$, so that $F^{\mu \nu}$ in (1.2) is replaced by $H^{\mu \nu}:=g^{P-1 \mu \alpha} g^{P-1 \nu \beta} F_{\alpha \beta}$. The electric and magnetic permeabilities are then composed from the components of $g_{\mu \nu}^{P}$, cf [18,21-23]. In the simplest case, one obtains as material equations the well known proportionalities between the field strengths and inductions. The second possibility, considered in this paper, are inductions depending on the preceding time evolution of the field strengths. In Fourier space, this results in frequencydependent permeabilities, and in real space the material equations become linear integral 
equations with regard to time, with the permeabilities as kernels (cf section 4). Frequencydependent permeabilities always result in nonlinear dispersion relations, i.e. in a nonlinear dependence of the wavevector on the frequency. Thus, in the case of electromagnetic fields, one cannot assume a frequency-dependent permeability for spacetime, as this would lead to a broadening of spectral lines [24]. Unlike in electrodynamics, there does not exist a proper retarded Green function for tachyons, the only Green function supported outside the lightcone is time-symmetric [14,25], which suggests invoking the ether as an absorber, to turn advanced modes of time-symmetric fields into retarded ones, quite similarly to the Wheeler-Feynman theory [26]. This is the reason for considering frequency-dependent permeabilities for the Proca field. In the case of tachyons, the dispersion relation is already nonlinear due to the mass-square, and frequency-dependent permeabilities are permissible, as long as they do not affect electromagnetic fields.

I conclude this introduction with an outline of the underlying spacetime concept and explain how it relates to relativity principles and causality. Maxwell's equations are timesymmetric, so that for every causal retarded solution there exists an acausal counterpart, obtained by applying the advanced Green function to the respective charge and current distribution. However, causality is easily preserved just by ignoring the advanced Green function, in fact, the causality principle is usually invoked to justify that. We adopt the commonly accepted causality: every effect has a cause, the cause precedes the effect and the distinction of cause and effect is unambiguous. Within the same causality, Wheeler and Feynman suggested a different way of explaining the apparent non-existence of advanced electromagnetic radiation [26]. They assumed an absorber, a collective response of the cosmic electric charges to the advanced component of the time-symmetric (half-retarded, halfadvanced) Green function. Instead of appealing to causality, they tried to explain it in terms of a cosmic absorber that turns advanced modes into retarded ones. The absorber generates the second half of the retarded Green function and wipes out the advanced component of the initial time-symmetric one.

As mentioned, in electrodynamics advanced wave modes can be discarded on the grounds of the causality principle alone, without considering a cosmic absorber in local radiation problems. The notion of advanced and retarded is relativistically invariant for (sub-)luminal wave fields, and so advanced solutions can be consistently ignored in a relativistic setting. Hence, in electrodynamics, one can either eliminate advanced solutions by invoking the causality principle, or try to explain the lack of advanced radiation by means of a cosmic absorber. If opting for the latter, the causality principle emerges from the fact that local physical systems can only within limits be thought of as being detached from the rest of the universe. In effect, however, the Wheeler-Feynman theory does not really alter electrodynamics, apart from putting it into a cosmological perspective, it leads to the same results as vacuum electrodynamics.

In electromagnetic theory, there is no real necessity to consider an absorber, but this changes for superluminal wave propagation. The advanced and retarded components of tachyonic wave fields are no longer invariant, because Lorentz boosts can change the time order of events connected by superluminal signals, that is, of events with a spacelike separation [5-9]. Therefore, one cannot define a retarded or advanced superluminal Green function, and a wave field retarded in one rest frame may appear advanced in another. If we assume the relativity principle, causality cannot be achieved simply by discarding advanced solutions, because there is no relativistically invariant way to even distinguish between retarded and advanced. So we are left with the second option, an absorber. We will show that in the rest frame of the absorber superluminal radiation is strictly retarded, by the conversion of advanced modes described above, starting with the time-symmetric Green function. In an expanding 
spacetime, the absorber frame can be identified with the comoving Robertson-Walker frame, but initially we will consider a Minkowskian universe, and identify the absorber frame with the static galaxy grid.

In the absorber frame there are no advanced modes, but retarded modes may appear advanced in the proper time of observers moving in the galaxy grid. Likewise, an outgoing wave train in the absorber frame can appear as a superposition of outgoing and incoming waves in the observer's rest frame. This happens when the cosmic time order of the absorber frame is inverted by the Lorentz boost (cf [16,25] for examples and a discussion of tachyonic energy in the geometrical optics limit). Every observer can compare his proper time with cosmic time and come to unambiguous conclusions concerning causality, emission and absorption. In the absorber frame tachyonic wave propagation is always retarded, and it serves as reference frame for all observers.

The cause precedes the effect in cosmic time. In the case of superluminal signal transfer, this distinguishes cosmic time from the proper time of observers moving in the galaxy grid and turns the absorber frame into an absolute spacetime. This is in striking contrast to subluminal and electromagnetic wave propagation, where the comoving galaxy frame is a mere matter of convenience when dealing with the galaxy recession and all that goes with it [24]. However, otherwise cosmic time in electromagnetic theory is not preferred to any other (e.g. locally geodesic) time coordinate, as the time order in timelike connections is preserved and so is retardation. In the context of tachyonic wave propagation, the comoving absorber frame is not just more useful than any other frame for cosmological reasoning, it constitutes the absolute cosmic spacetime - the universal frame of reference for all observers moving in the galaxy grid.

The causality principle and the relativity principle are mutually inconsistent if events are connected by superluminal signals, as Lorentz boosts can change the time order of events with a spacelike connection. A relativity principle based on Lorentz transformations in locally geodesic neighbourhoods and imposed on superluminal motion unavoidably conflicts with causality $[8,9,16]$. Causality suggests considering superluminal signals in the context of an absolute spacetime, and the cosmic absorber just provides that. I also note that relativity theory is a theory of subluminal motion, and no inferences about the existence of superluminal signals can be drawn from it. Apart from causality, the absorber also supplies the medium for wave propagation, sought for by the electricians of the 18th and 19th centuries [17]. We will define the ether microscopically and quite quantitatively by oscillating tachyonic charges responsive to the Proca field, uniformly distributed over space. These charges convert the advanced tachyonic modes into retarded ones, essentially in the same way as the electric charges in the Wheeler-Feynman theory do.

In section 2 we sketch the Proca equation in Minkowski space, introduce the field strengths and the Maxwell equations for negative mass-square, the time-symmetric Green function, as well as time-symmetric wave fields generated by subluminal currents. We show that time-symmetric superluminal fields can be decomposed into retarded and advanced modes independently satisfying the field equations, and discuss the energy density of transverse and longitudinal tachyon fields as well as the radiant power of their sources. In section 3 we assemble the tachyonic Liénard-Wiechert potentials, i.e. we calculate the asymptotic superluminal radiation fields of classical subluminal point particles. The tachyonic radiation of oscillating charges is investigated (spectral density, intensity, cross sections) and compared to electromagnetic radiation.

In section 4 we study the Proca equation with negative mass-square in a permeable spacetime with homogeneous and isotropic, though frequency-dependent and absorptive permeabilities. We derive the material equations in analogy with electrodynamics, and study 
the geometrical optics limit as well as the Poynting theorem, that is, the energy balance for transversal and longitudinal tachyon radiation in a refractive and absorptive spacetime. A classical oscillator model for the ether is introduced.

In section 5 we discuss the Wheeler-Feynman theory in the described context, for a time-symmetric tachyonic Proca field propagating in the permeable spacetime. We derive the superluminal absorber field generated by the oscillators of the ether, and show how retarded tachyon fields arise from subluminal currents. In section 6 we present our conclusions, and in the appendix we list a complete set of singular functions for wave propagation with negative mass-square.

\section{Superluminal radiation fields}

The Proca equation [27] with negative mass-square,

$$
F_{, v}^{\mu \nu}-m_{\mathrm{t}}^{2} A^{\mu}=c^{-1} j^{\mu},
$$

$\left(m_{\mathrm{t}}>0\right.$ in our notation) can equivalently be written as

$$
\left(\square+m_{\mathrm{t}}^{2}\right) A_{\mu}=-c^{-1} j_{\mu}, \quad A_{, \mu}^{\mu}=0,
$$

with the d'Alembertian $\square:=\eta^{\mu \nu} \partial_{\mu} \partial_{\nu}, \eta_{\mu \nu}=\operatorname{diag}\left(-c^{2}, 1,1,1\right)$. The Lorentz condition is a consequence of (2.1) and current conservation. $m_{\mathrm{t}}$ has the dimension of an inverse length, and is meant as a shortcut for $m_{\mathrm{t}} c / \hbar, \operatorname{cf}(1.2) .\left(m_{\mathrm{t}} / m_{\mathrm{e}} \approx \frac{1}{238}\right.$, estimated from Lamb shifts in hydrogenic systems $[13,14]$.) Tachyonic $E$ and $B$ fields are related to the vector potential by

$$
E_{i}=c^{-1} F_{i 0}=c^{-1}\left(\nabla A_{0}-\partial \boldsymbol{A} / \partial t\right), \quad B^{k}=\frac{1}{2} \varepsilon^{k i j} F_{i j}=\operatorname{rot} \boldsymbol{A} .
$$

The field equations (2.1) or (2.2) decompose into Maxwell's equations,

$$
\begin{array}{rlrl}
\operatorname{div} \boldsymbol{B} & =0, & & \operatorname{rot} \boldsymbol{E}+c^{-1} \partial \boldsymbol{B} / \partial t=0, \\
\operatorname{div} \boldsymbol{E}=\rho-c^{-1} m_{\mathrm{t}}^{2} A_{0}, & & \operatorname{rot} \boldsymbol{B}-c^{-1} \partial \boldsymbol{E} / \partial t=c^{-1} \boldsymbol{j}+m_{\mathrm{t}}^{2} \boldsymbol{A},
\end{array}
$$

where we have identified $j^{\mu}=(\rho, j)$. The vector potential is completely determined by the current and the $\boldsymbol{E}$ and $\boldsymbol{B}$ fields, thanks to the tachyon mass. The classical energy density and the Poynting vector are readily found as

$$
\begin{aligned}
& \rho_{E}=\frac{1}{2}\left(\boldsymbol{E}^{2}+\boldsymbol{B}^{2}\right)-\left(m_{\mathrm{t}}^{2} / 2\right)\left(c^{-2} A_{0} A_{0}+\boldsymbol{A}^{2}\right), \\
& \boldsymbol{S}=c \boldsymbol{E} \times \boldsymbol{B}+m_{t}^{2} A_{0} \boldsymbol{A}, \quad \operatorname{div} \boldsymbol{S}+\partial \rho_{E} / \partial t=0 .
\end{aligned}
$$

The inversion of the wave equation (2.2) is effected by

$$
A_{\alpha}(x)=\int_{R^{4}} G\left(x-x^{\prime}\right) j_{\alpha}\left(x^{\prime}\right) \mathrm{d} x^{\prime}, \quad\left(\square+m_{\mathrm{t}}^{2}\right) G(t, x)=-c^{-1} \delta(t) \delta(\boldsymbol{x}) ;
$$

the Lorentz condition is evidently satisfied by this ansatz. In the case of photons or subluminal particles ( $m_{\mathrm{t}}^{2} \leqslant 0$ in our notation), one would choose for $G$ the retarded Green function, supported on the forward lightcone. One expects a classical Green function for tachyons to be supported outside the lightcone (at least as long as we do not consider curvature effects), as tachyons cannot move below the speed of light. The complete solution of (2.7) is obtained by analytic continuation, $m \rightarrow \pm \mathrm{i} m$, of the subluminal Green functions (retarded, advanced, Feynman and Dyson propagators), cf the appendix. The only linear combination of these continuations vanishing inside the cone is

$$
G^{\mathrm{sym}}(t, \boldsymbol{x})=\frac{1}{4 \pi} \delta\left(r^{2}-c^{2} t^{2}\right)-\frac{m_{\mathrm{t}}}{8 \pi} \theta\left(r^{2}-c^{2} t^{2}\right) \frac{J_{1}\left(m_{\mathrm{t}} \sqrt{r^{2}-c^{2} t^{2}}\right)}{\sqrt{r^{2}-c^{2} t^{2}}} .
$$


The limit $m_{\mathrm{t}} \rightarrow 0$ is smooth, $J_{1}(z) \sim z / 2$. The distribution (2.8) admits a very handy Fourier transform,

$$
\begin{aligned}
& \int_{-\infty}^{+\infty} G^{\mathrm{sym}}(t, \boldsymbol{x}) \mathrm{e}^{\mathrm{i} \omega t} \mathrm{~d} t=\frac{1}{4 \pi c} \frac{1}{r} \cos (k(\omega) r), \quad k:=\sqrt{\omega^{2} / c^{2}+m_{\mathrm{t}}^{2}}, \\
& G^{\mathrm{sym}}(t, \boldsymbol{x})=\frac{1}{4 \pi^{2} c r} \int_{0}^{\infty} \mathrm{d} \omega \mathrm{e}^{-\varepsilon k(\omega)} \cos (\omega t) \cos (k(\omega) r) .
\end{aligned}
$$

$(k(\omega)>0$.) In (2.10) we have inserted an $\varepsilon$-regularizer, chosen in such a way that the $\omega$-integral is solvable in closed form, via a standard representation of Bessel functions [28],

$$
\int_{0}^{\infty} \mathrm{d} \omega \cos (\omega t) \exp (-(\varepsilon-\mathrm{i} r) k(\omega))=-\mathrm{i} c m_{\mathrm{t}} r \lambda_{\varepsilon}^{-1 / 2} K_{1}\left(m_{\mathrm{t}} \lambda_{\varepsilon}^{1 / 2}\right)
$$

with $\lambda_{\varepsilon}:=c^{2} t^{2}+(\varepsilon-\mathrm{i} r)^{2}$. Equality in (2.9)-(2.11) is meant distributionally, we have already dropped terms not contributing in the limit $\varepsilon \rightarrow 0$. The identity of (2.10) with (2.8) is easily seen via (2.11); we extract the pole part of $K_{1}(z)=z^{-1}+\mathrm{O}(z \log z)$, and then apply analytic continuation, $\mathrm{cf}(\mathrm{A} .4)$, as well as the identity $\mp \pi \mathrm{i} \delta(x)=(x \pm \mathrm{i} \varepsilon)^{-1}-P x^{-1}$. Though there does not exist a proper retarded Green function outside the lightcone, it is still possible to generate retarded wave fields by convolutions with the current, cf (5.13)-(5.18).

Next, we represent the spatial component of the vector potential as

$$
\boldsymbol{A}(\boldsymbol{x}, t)=\frac{1}{2 \pi} \int_{0}^{\infty}\left(\hat{\boldsymbol{A}}(\boldsymbol{x}, \omega) \mathrm{e}^{-\mathrm{i} \omega t}+\text { c.c. }\right) \mathrm{d} \omega, \quad \hat{\boldsymbol{A}}(\boldsymbol{x}, \omega)=\int_{-\infty}^{+\infty} \boldsymbol{A}(\boldsymbol{x}, t) \mathrm{e}^{\mathrm{i} \omega t} \mathrm{~d} t,
$$

so that $\hat{\boldsymbol{A}}^{*}(\boldsymbol{x}, \omega)=\hat{\boldsymbol{A}}(\boldsymbol{x},-\omega)$, and analogous relations hold for the time component, the charge and current densities, and the $E$ and $B$ fields. If we consider monochromatic waves, we will define $\boldsymbol{A}=2 \operatorname{Re}\left(\hat{\boldsymbol{A}} \mathrm{e}^{-\mathrm{i} \omega t}\right)$; this convention, twice the real part, is used throughout this paper. The Fourier amplitude $\hat{\boldsymbol{A}}(\boldsymbol{x}, \omega)$ can be split into $\hat{\boldsymbol{A}}=\mathrm{e}^{\mathrm{i} \alpha}\left(\boldsymbol{a}_{1}+\mathrm{i} \boldsymbol{a}_{2}\right)$, with orthogonal real vectors $a_{1,2}$; in the case of linear polarization, $\hat{\boldsymbol{A}}$ is a real vector up to a phase factor. We write $\hat{\boldsymbol{A}}^{2}:=\hat{\boldsymbol{A}} \cdot \hat{\boldsymbol{A}}$ and $|\hat{\boldsymbol{A}}|^{2}:=\hat{\boldsymbol{A}} \cdot \hat{\boldsymbol{A}}^{*}$ for complex 3 -vectors; unit vector normalization will only be applied to real vectors. Fourier modes with regard to time will always be defined as in (2.12), for all fields, and denoted by a hat.

The Maxwell equations in Fourier space are obtained by substituting $\partial / \partial t \rightarrow-i \omega$ into (2.4), and by replacing the fields by their Fourier components $\hat{\boldsymbol{A}}(\boldsymbol{x}, \omega), \hat{\boldsymbol{E}}(\boldsymbol{x}, \omega)$, etc; this will be discussed in greater detail in section 4 , when we consider frequency-dependent permeabilities. Lorentz condition and current conservation read in Fourier space as

$$
\mathrm{i} \omega \hat{A}_{0}(\boldsymbol{x}, \omega)=-c^{2} \operatorname{div} \hat{\boldsymbol{A}}(\boldsymbol{x}, \omega), \quad \mathrm{i} \omega \hat{\rho}(\boldsymbol{x}, \omega)=\nabla \cdot \hat{\boldsymbol{j}}(\boldsymbol{x}, \omega) .
$$

We find, via (2.7) and (2.9), $\hat{\boldsymbol{A}}(\boldsymbol{x}, \omega)=\hat{\boldsymbol{A}}^{+}+\hat{\boldsymbol{A}}^{-}$, with

$$
\hat{\boldsymbol{A}}^{ \pm}(\boldsymbol{x}, \omega):=\frac{1}{8 \pi c} \int \mathrm{d} \boldsymbol{x}^{\prime} \hat{\boldsymbol{j}}\left(\boldsymbol{x}^{\prime}, \omega\right) \frac{\exp \left( \pm \mathrm{i} k(\omega)\left|\boldsymbol{x}-\boldsymbol{x}^{\prime}\right|\right)}{\left|\boldsymbol{x}-\boldsymbol{x}^{\prime}\right|} ;
$$

the time components $\hat{A}_{0}^{ \pm}(\boldsymbol{x}, \omega)$ follow from (2.14) by the substitution $\hat{\boldsymbol{j}}\left(\boldsymbol{x}^{\prime}, \omega\right) \rightarrow-c^{2} \hat{\rho}\left(\boldsymbol{x}^{\prime}, \omega\right)$, or from (2.13). ( $\hat{j}$ and $\hat{\rho}$ are the Fourier transforms of $j$ and $\rho$ as defined in (2.12).) Equation (2.14) holds only for positive frequencies, otherwise we have to replace $k(\omega)$ by $\operatorname{sign}(\omega) k(\omega)$ or by $k=\omega n_{0} / c$, with $n_{0}:=\sqrt{1+\left(m_{\mathrm{t}} c / \omega\right)^{2}}$; we will return to that in (4.4) and (5.2). The retarded and advanced wave fields $A_{\mu}^{\text {ret/adv }}=2 A_{\mu}^{+/-}$are exact solutions of the field equations (2.1) or (2.2), because $\left(\Delta+k^{2}\right)\left(r^{-1} \sin (k r)\right) \equiv 0$.

By expanding $\left|\boldsymbol{x}-\boldsymbol{x}^{\prime}\right|=r-\boldsymbol{n} \cdot \boldsymbol{x}^{\prime}+\mathrm{O}(1 / r)$, with $\boldsymbol{n}:=\boldsymbol{x} / r$, we obtain

$$
\hat{\boldsymbol{A}}^{\mathrm{ret} / \mathrm{adv}}(\boldsymbol{x}, \omega) \sim \frac{1}{4 \pi c} \frac{\mathrm{e}^{ \pm \mathrm{i} k r}}{r} \hat{\boldsymbol{J}}^{ \pm}(\boldsymbol{x}, \omega), \quad \hat{\boldsymbol{J}}^{ \pm}:=\int \mathrm{d} \boldsymbol{x}^{\prime} \hat{\boldsymbol{j}}\left(\boldsymbol{x}^{\prime}, \omega\right) \exp \left(\mp \mathrm{i} k \boldsymbol{n} \cdot \boldsymbol{x}^{\prime}\right) .
$$


The asymptotic transversal and longitudinal components are found if we replace $\hat{j}$ in $(2.15)$ by $\hat{\boldsymbol{j}}^{\mathrm{T}}\left(\boldsymbol{x}^{\prime}, \boldsymbol{x}\right):=\hat{\boldsymbol{j}}\left(\boldsymbol{x}^{\prime}\right)-\boldsymbol{n}\left(\boldsymbol{n} \cdot \hat{\boldsymbol{j}}\left(\boldsymbol{x}^{\prime}\right)\right)$ or $\hat{\boldsymbol{j}}^{\mathrm{L}}\left(\boldsymbol{x}^{\prime}, \boldsymbol{x}\right):=\boldsymbol{n}\left(\boldsymbol{n} \cdot \hat{\boldsymbol{j}}\left(\boldsymbol{x}^{\prime}\right)\right)$, respectively.

In the following we will only consider retarded fields, writing $\hat{\boldsymbol{J}}$ for $\hat{\boldsymbol{J}}^{+}$and $\hat{\boldsymbol{A}}$ for $\hat{\boldsymbol{A}}^{\text {ret }}$; in section 5 we will return to advanced solutions. We obtain the components of the retarded vector potential as

$\hat{\boldsymbol{A}}^{\mathrm{T}, \mathrm{L}}(\boldsymbol{x}, \omega) \sim \frac{1}{4 \pi c} \frac{\mathrm{e}^{\mathrm{i} k r}}{r} \hat{\boldsymbol{J}}^{\mathrm{T}, \mathrm{L}}(\boldsymbol{x}, \omega), \quad \hat{\boldsymbol{J}}^{\mathrm{T}}:=\hat{\boldsymbol{J}}-\boldsymbol{n}(\boldsymbol{n} \cdot \hat{\boldsymbol{J}}), \quad \hat{\boldsymbol{J}}^{\mathrm{L}}:=\boldsymbol{n}(\boldsymbol{n} \cdot \hat{\boldsymbol{J}})$.

When differentiating (2.16) with respect to $x$, it is sufficient to take only the $r$ dependence of $\mathrm{e}^{\mathrm{i} k r}$ into account. We so find, $\operatorname{cf}(2.3),(2.12)$ and (2.13),

$$
\begin{aligned}
& \hat{\boldsymbol{E}}^{\mathrm{T}}(\boldsymbol{x}, \omega) \sim \mathrm{i} c^{-1} \omega \hat{\boldsymbol{A}}^{\mathrm{T}}, \quad \hat{\boldsymbol{B}}^{\mathrm{T}} \sim \mathrm{i} k(\omega) \boldsymbol{n} \times \hat{\boldsymbol{A}}^{\mathrm{T}}, \quad \hat{A}_{0}^{\mathrm{T}}=\mathrm{O}\left(1 / r^{2}\right), \\
& \hat{\boldsymbol{E}}^{\mathrm{L}} \sim-\mathrm{i} \frac{m_{\mathrm{t}}^{2} c}{\omega} \hat{\boldsymbol{A}}^{\mathrm{L}}, \quad \hat{\boldsymbol{B}}^{\mathrm{L}} \sim \mathrm{O}\left(1 / r^{2}\right), \quad \hat{A}_{0}^{\mathrm{L}} \sim-c^{2} \frac{k(\omega)}{\omega} \boldsymbol{n} \cdot \hat{\boldsymbol{A}}^{\mathrm{L}},
\end{aligned}
$$

with $\hat{\boldsymbol{A}}^{\mathrm{T}, \mathrm{L}}(\boldsymbol{x}, \omega)$ in $(2.16)$.

The energy density in (2.5) is positive for transversal modes and negative for longitudinal ones. Accordingly, we define the energy-momentum tensor for longitudinal modes with opposite sign. The fields $\boldsymbol{E}^{\mathrm{T}, \mathrm{L}}(\boldsymbol{x}, t)$, etc relate to their Fourier components (2.17) and (2.18) as defined in (2.12), and we find in leading asymptotic order the energy densities and flux vectors as

$$
\begin{array}{ll}
\rho_{\mathrm{E}}^{\mathrm{T}}(\boldsymbol{x}, t) \sim \frac{1}{2}\left(\boldsymbol{E}^{\mathrm{T} 2}+\boldsymbol{B}^{\mathrm{T} 2}-m_{\mathrm{t}}^{2} \boldsymbol{A}^{\mathrm{T} 2}\right), & \boldsymbol{S}^{\mathrm{T}} \sim c \boldsymbol{E}^{\mathrm{T}} \times \boldsymbol{B}^{\mathrm{T}}, \\
\rho_{\mathrm{E}}^{\mathrm{L}} \sim \frac{1}{2} m_{\mathrm{t}}^{2}\left(A_{0}^{\mathrm{L} 2}+\boldsymbol{A}^{\mathrm{L} 2}\right)-\frac{1}{2} \boldsymbol{E}^{\mathrm{L} 2}, & \boldsymbol{S}^{\mathrm{L}} \sim-m_{\mathrm{t}}^{2} A_{0}^{\mathrm{L}} \boldsymbol{A}^{\mathrm{L}} .
\end{array}
$$

In the following we will give a quantitative discussion of the energy concept for classical superluminal wave propagation, which is also the main topic of sections 3 and 4 . At first we discuss the radiant energy of a tachyonic charge. The time-integrated flux, based on (2.17)(2.20), is

$$
\begin{aligned}
& \int_{-\infty}^{+\infty} \boldsymbol{S}^{\mathrm{T}} \mathrm{d} t \sim \frac{\boldsymbol{n}}{\pi} \int_{0}^{\infty} \omega k(\omega)\left|\hat{\boldsymbol{A}}^{\mathrm{T}}\right|^{2} \mathrm{~d} \omega \\
& \int_{-\infty}^{+\infty} \boldsymbol{S}^{\mathrm{L}} \mathrm{d} t \sim m_{\mathrm{t}}^{2} c^{2} \frac{\boldsymbol{n}}{\pi} \int_{0}^{\infty} \frac{k(\omega)}{\omega}\left|\hat{\boldsymbol{A}}^{\mathrm{L}}\right|^{2} \mathrm{~d} \omega
\end{aligned}
$$

The energy flux per unit time through the surface element $r^{2} \mathrm{~d} \Omega$ (sufficiently distant from the support of the current centred at the coordinate origin) is $\mathrm{d} I=\boldsymbol{S} \cdot \boldsymbol{n} \boldsymbol{r}^{2} \mathrm{~d} \Omega$. The energy density can be extracted from $(2.21)$,

$$
\begin{aligned}
& \int_{-\infty}^{+\infty} \boldsymbol{S}^{\mathrm{T}, \mathrm{L}} \cdot \boldsymbol{n} \mathrm{d} t=\int_{0}^{\infty} \mathrm{d} \hat{E}^{\mathrm{T}, \mathrm{L}}(\omega), \\
& \mathrm{d} \hat{E}^{\mathrm{T}}(\omega):=\frac{1}{\pi} \omega k(\omega)\left|\hat{\boldsymbol{A}}^{\mathrm{T}}\right|^{2} \mathrm{~d} \omega, \quad \mathrm{d} \hat{E}^{\mathrm{L}}(\omega):=\frac{1}{\pi} \frac{m_{\mathrm{t}}^{2} c^{2}}{\omega} k(\omega)\left|\hat{\boldsymbol{A}}^{\mathrm{L}}\right|^{2} \mathrm{~d} \omega,
\end{aligned}
$$

so that the spectral density of the total radiated energy is found to be

$$
\mathrm{d} E^{\mathrm{T}, \mathrm{L}}(\omega):=\lim _{r \rightarrow \infty} \int_{|x|=r} \mathrm{~d} \hat{E}^{\mathrm{T}, \mathrm{L}}(\omega) r^{2} \mathrm{~d} \Omega .
$$

These formulae are quite general, but some restrictions do apply. The current has to be localized around the coordinate origin, uniformly in time (which is assumed in the approximation made 
in (2.15)) and the Fourier transforms $\hat{\boldsymbol{A}}^{\mathrm{T}, \mathrm{L}}(\boldsymbol{x}, \omega)$ should not be excessively singular, so that they can be squared. However, in (2.15)-(2.23) there is not yet a dipole approximation involved, so there is no restriction on the wavelength.

We study this energy concept for monochromatic fields, $\boldsymbol{A}^{\mathrm{T}, \mathrm{L}}(\boldsymbol{x}, t)=\hat{\boldsymbol{A}}^{\mathrm{T}, \mathrm{L}}(\boldsymbol{x}, \omega) \mathrm{e}^{-\mathrm{i} \omega t}+$ c.c., with $\hat{\boldsymbol{A}}^{\mathrm{T}, \mathrm{L}}$ as defined in (2.16). The energy density and the flux of a single retarded mode is calculated via (2.17)-(2.20). Time-averaged over a period of $2 \pi / \omega$,

$\left\langle\rho_{\mathrm{E}}^{\mathrm{T}}\right\rangle \sim 2 \frac{\omega^{2}}{c^{2}}\left|\hat{\boldsymbol{A}}^{\mathrm{T}}\right|^{2}, \quad\left\langle\rho_{\mathrm{E}}^{\mathrm{L}}\right\rangle \sim 2 m_{\mathrm{t}}^{2}\left|\hat{\boldsymbol{A}}^{\mathrm{L}}\right|^{2}, \quad\left\langle\boldsymbol{S}^{\mathrm{T}, \mathrm{L}}\right\rangle \sim c^{2} \frac{k(\omega)}{\omega}\left\langle\rho_{\mathrm{E}}^{\mathrm{T}, \mathrm{L}}\right\rangle \boldsymbol{n}$.

The dipole approximation amounts to the substitution $\hat{\boldsymbol{J}}^{\mathrm{T}, \mathrm{L}} \rightarrow \hat{\boldsymbol{J}}_{d}^{\mathrm{T}, \mathrm{L}}$ in (2.16),

$\hat{\boldsymbol{J}}_{d}^{\mathrm{T}, \mathrm{L}}:=\int \mathrm{d} \boldsymbol{x}^{\prime} \hat{\boldsymbol{j}}^{\mathrm{T}, \mathrm{L}}\left(\boldsymbol{x}^{\prime}, \boldsymbol{x}, \omega\right)=-\mathrm{i} \omega \hat{\boldsymbol{d}}^{\mathrm{T}, \mathrm{L}}(\boldsymbol{x}, \omega), \quad \hat{\boldsymbol{d}}(\omega):=\int \boldsymbol{x} \hat{\rho}(\boldsymbol{x}, \omega) \mathrm{d} \boldsymbol{x}$,

with the usual projections $\hat{\boldsymbol{d}}^{\mathrm{T}}:=\hat{\boldsymbol{d}}-\boldsymbol{n}(\boldsymbol{n} \cdot \hat{\boldsymbol{d}})$ and $\hat{\boldsymbol{d}}^{\mathrm{L}}:=\boldsymbol{n}(\boldsymbol{n} \cdot \hat{\boldsymbol{d}})$. In this approximation, the energy transversally and longitudinally radiated per unit time reads

$$
\begin{aligned}
& \int\left\langle\boldsymbol{S}^{\mathrm{T}} \cdot \boldsymbol{n}\right\rangle r^{2} \mathrm{~d} \Omega \sim \frac{1}{3 \pi} \frac{\omega^{3} k(\omega)}{c^{2}}|\hat{\boldsymbol{d}}(\omega)|^{2}, \\
& \int\left\langle\boldsymbol{S}^{\mathrm{L}} \cdot \boldsymbol{n}\right\rangle r^{2} \mathrm{~d} \Omega \sim \frac{1}{6 \pi} m_{\mathrm{t}}^{2} \omega k(\omega)|\hat{\boldsymbol{d}}(\omega)|^{2},
\end{aligned}
$$

respectively. An explicit discussion of the spectral energy densities in dipole approximation will be given in the next section.

\section{Tachyonic Liénard-Wiechert potentials}

We consider the trajectory of a subluminal particle $x_{0}(t), \boldsymbol{v}=\dot{\boldsymbol{x}}_{0}$, carrying tachyonic charge $q$, so that $j^{0}=\rho=q \delta\left(\boldsymbol{x}-\boldsymbol{x}_{0}(t)\right), \boldsymbol{j}=q \boldsymbol{v} \delta\left(\boldsymbol{x}-\boldsymbol{x}_{0}(t)\right)$, and

$$
(\hat{\rho}, \hat{\boldsymbol{j}})(\boldsymbol{x}, \omega)=q \int_{-\infty}^{+\infty}(1, \boldsymbol{v}(t)) \delta\left(\boldsymbol{x}-\boldsymbol{x}_{0}(t)\right) \mathrm{e}^{\mathrm{i} \omega t} \mathrm{~d} t .
$$

The time-symmetric potential generated by this current reads, $\mathrm{cf}(2.7)$,

$$
\left(A_{0}, \boldsymbol{A}\right)(\boldsymbol{x}, t)=q \int_{-\infty}^{+\infty}\left(-c^{2}, \boldsymbol{v}\left(t^{\prime}\right)\right) G^{\mathrm{sym}}\left(\boldsymbol{x}-\boldsymbol{x}_{0}\left(t^{\prime}\right), t-t^{\prime}\right) \mathrm{d} t^{\prime} .
$$

The asymptotic retarded and advanced potentials are thus given by (2.15), with

$$
\hat{\boldsymbol{J}}^{ \pm}(\boldsymbol{x}, \omega)=q \int_{-\infty}^{+\infty} \mathrm{d} t^{\prime} \boldsymbol{v}\left(t^{\prime}\right) \exp \left[\mathrm{i}\left(\omega t^{\prime} \mp k(\omega) \boldsymbol{n} \cdot \boldsymbol{x}_{0}\left(t^{\prime}\right)\right)\right]
$$

where $\boldsymbol{n}=\boldsymbol{x} / r$. We will only consider retarded fields, writing $\hat{\boldsymbol{J}}$ for $\hat{\boldsymbol{J}}^{+}$. The transversal and longitudinal components $\hat{\boldsymbol{J}}^{\mathrm{T}, \mathrm{L}}$ are defined by the projections $\boldsymbol{v}^{\mathrm{T}}:=\boldsymbol{v}-\boldsymbol{n}(\boldsymbol{n} \cdot \boldsymbol{v})$ and $\boldsymbol{v}^{\mathrm{L}}:=\boldsymbol{n}(\boldsymbol{n} \cdot \boldsymbol{v})$, respectively, substituted for $\boldsymbol{v}$ into (3.3). We will focus on the dipole approximation, which means to drop the $\boldsymbol{n} \cdot \boldsymbol{x}_{0}\left(t^{\prime}\right)$-term in (3.3), so that, $\operatorname{cf}(2.16)$,

$$
\begin{array}{ll}
\hat{\boldsymbol{J}}^{\mathrm{T}, \mathrm{L}}(\boldsymbol{x}, \omega) \sim q \hat{\boldsymbol{v}}^{\mathrm{T}, \mathrm{L}}, & \hat{\boldsymbol{A}}^{\mathrm{T}, \mathrm{L}}(\boldsymbol{x}, \omega) \sim \frac{q}{4 \pi c} \frac{\mathrm{e}^{\mathrm{i} k r}}{r} \hat{\boldsymbol{v}}^{\mathrm{T}, \mathrm{L}}(\boldsymbol{x}, \omega), \\
\hat{\boldsymbol{v}}(\omega):=\int_{-\infty}^{+\infty} \boldsymbol{v}(t) \mathrm{e}^{\mathrm{i} \omega t} \mathrm{~d} t, & \hat{\boldsymbol{v}}^{\mathrm{T}}:=\hat{\boldsymbol{v}}-\boldsymbol{n}(\boldsymbol{n} \cdot \hat{\boldsymbol{v}}),
\end{array}
$$


the Fourier transforms of the other fields are then given by (2.17) and (2.18), and their realspace expressions are obtained via (2.12). The spectral energy densities are readily assembled from (2.22) and (2.23),

$\mathrm{d} E^{\mathrm{T}}(\omega)=\frac{q^{2}}{6 \pi^{2} c^{2}} \omega k(\omega)|\hat{\boldsymbol{v}}(\omega)|^{2} \mathrm{~d} \omega, \quad \mathrm{d} E^{\mathrm{L}}(\omega)=\frac{1}{2} \frac{m_{\mathrm{t}}^{2} c^{2}}{\omega^{2}} \mathrm{~d} E^{\mathrm{T}}(\omega)$,

which relate to the dipole $\boldsymbol{d}(t):=q \boldsymbol{r}(t)$ via $q \hat{\boldsymbol{v}}(\omega)=-\mathrm{i} \omega \hat{\boldsymbol{d}}(\omega)$.

We consider a monochromatic wave, $\hat{\boldsymbol{E}}(\tilde{\omega}) \mathrm{e}^{-\mathrm{i} \tilde{\omega} t}+$ c.c., $\tilde{\omega}>0$, and a damped oscillator coupled to this mode, $\boldsymbol{r}=\hat{\boldsymbol{r}}+\hat{\boldsymbol{r}}^{*}$,

$$
\hat{\boldsymbol{r}}^{\prime \prime}+\gamma_{0} \hat{\boldsymbol{r}}^{\prime}+\omega_{0}^{2} \hat{\boldsymbol{r}}=\frac{q_{0}}{m_{0}} \hat{\boldsymbol{E}} \mathrm{e}^{-\mathrm{i} \tilde{\omega} t}, \quad \hat{\boldsymbol{r}}=\frac{1}{\omega_{0}^{2}-\tilde{\omega}^{2}-\mathrm{i} \gamma_{0} \tilde{\omega}} \frac{q_{0}}{m_{0}} \hat{\boldsymbol{E}} \mathrm{e}^{-\mathrm{i} \tilde{\omega} t},
$$

where $\omega_{0}$ is the free oscillator frequency, $\gamma_{0}$ the positive damping constant, $m_{0}$ the mass and $q_{0}$ the tachyonic charge of the oscillator. We assume for simplicity $\hat{\boldsymbol{E}}(\tilde{\omega})$ to be real (longitudinal or linear transversal polarization), and neglect the spatial variation of $\hat{\boldsymbol{E}}(\tilde{\omega})$, which means that the oscillator size is supposed to be much smaller than the wavelength of the tachyon radiation. (There is a maximal wavelength for tachyon radiation, the Compton wavelength, cf the end of this section.) The transversal and longitudinal components $\hat{\boldsymbol{E}}^{\mathrm{T}, \mathrm{L}}(\tilde{\omega})$ are defined like $\hat{\boldsymbol{v}}^{\mathrm{T}, \mathrm{L}}$ in (3.4). The Fourier transform of the velocity is readily calculated,

$$
\hat{\boldsymbol{v}}(\omega)=-2 \pi \mathrm{i} \frac{q_{0}}{m_{0}}\left(\frac{\tilde{\omega} \delta(\omega-\tilde{\omega})}{\omega_{0}^{2}-\tilde{\omega}^{2}-\mathrm{i} \gamma_{0} \tilde{\omega}}-\frac{\tilde{\omega} \delta(\omega+\tilde{\omega})}{\omega_{0}^{2}-\tilde{\omega}^{2}+\mathrm{i} \gamma_{0} \tilde{\omega}}\right) \hat{\boldsymbol{E}}
$$

As both $\omega$ and $\tilde{\omega}$ are positive, the second term in (3.7) does not contribute in the Fourier transform (2.12) and will be dropped. We so find from (3.4),

$$
\begin{aligned}
& \boldsymbol{A}^{\mathrm{T}, \mathrm{L}}(\boldsymbol{x}, t) \sim-\mathrm{i}\left(\boldsymbol{a}^{\mathrm{T}, \mathrm{L}}-\boldsymbol{a}^{\mathrm{T}, \mathrm{L} *}\right), \\
& \boldsymbol{a}^{\mathrm{T}, \mathrm{L}}(r, t):=\frac{q q_{0}}{4 \pi} \frac{1}{m_{0} c} \frac{1}{r} \frac{\tilde{\omega} \exp (\mathrm{i}(k(\tilde{\omega}) r-\tilde{\omega} t))}{\omega_{0}^{2}-\tilde{\omega}^{2}-\mathrm{i} \gamma_{0} \tilde{\omega}} \hat{\boldsymbol{E}}^{\mathrm{T}, \mathrm{L}},
\end{aligned}
$$

and the other fields read according to (2.17),

$$
\begin{array}{lll}
\boldsymbol{E}^{\mathrm{T}}(\boldsymbol{x}, t) \sim c^{-1} \tilde{\omega}\left(\boldsymbol{a}^{\mathrm{T}}+\boldsymbol{a}^{\mathrm{T} *}\right), & \boldsymbol{B}^{\mathrm{T}} \sim k(\tilde{\omega}) \boldsymbol{n} \times\left(\boldsymbol{a}^{\mathrm{T}}+\boldsymbol{a}^{\mathrm{T} *}\right), & A_{0}^{\mathrm{T}}=\mathrm{O}\left(1 / r^{2}\right), \\
\boldsymbol{E}^{\mathrm{L}} \sim-\frac{m_{\mathrm{t}}^{2} c}{\tilde{\omega}}\left(\boldsymbol{a}^{\mathrm{L}}+\boldsymbol{a}^{\mathrm{L} *}\right), & \boldsymbol{B}^{\mathrm{L}} \sim \mathrm{O}\left(1 / r^{2}\right), & A_{0}^{\mathrm{L}} \sim \mathrm{i} c^{2} \frac{k(\tilde{\omega})}{\tilde{\omega}} \boldsymbol{n} \cdot\left(\boldsymbol{a}^{\mathrm{L}}-\boldsymbol{a}^{\mathrm{L} *}\right) .
\end{array}
$$

The components of the flux vector, $\mathrm{cf}$ (2.19) and (2.20), time-averaged over a period of $2 \pi / \tilde{\omega}$, are readily compiled,

$$
\left\langle\boldsymbol{S}^{\mathrm{T}}\right\rangle \sim 2 \tilde{\omega} k(\tilde{\omega}) \boldsymbol{n}\left|\boldsymbol{a}^{\mathrm{T}}\right|^{2}, \quad\left\langle\boldsymbol{S}^{\mathrm{L}}\right\rangle \sim 2 m_{\mathrm{t}}^{2} c^{2} \tilde{\omega}^{-1} k(\tilde{\omega}) \boldsymbol{n}\left|\boldsymbol{a}^{\mathrm{L}}\right|^{2}
$$

and give the intensity $\mathrm{d} I^{\mathrm{T}, \mathrm{L}}=\left\langle\boldsymbol{S}^{\mathrm{T}, \mathrm{L}}\right\rangle \cdot \boldsymbol{n} \boldsymbol{r}^{2} \mathrm{~d} \Omega$ of the transversal and longitudinal radiation,

$$
\mathrm{d} I^{\mathrm{T}}=2 \frac{q^{2} q_{0}^{2}}{(4 \pi)^{2}} \frac{\hat{\boldsymbol{E}}^{2}}{m_{0}^{2} c^{2}} \frac{\tilde{\omega}^{3} k(\tilde{\omega}) \sin ^{2} \theta \mathrm{d} \Omega}{\left(\omega_{0}^{2}-\tilde{\omega}^{2}\right)^{2}+\left(\gamma_{0} \tilde{\omega}\right)^{2}}, \quad \frac{\mathrm{d} I^{\mathrm{L}}}{\mathrm{d} I^{\mathrm{T}}}=\frac{m_{\mathrm{t}}^{2} c^{2}}{\tilde{\omega}^{2}} \cot ^{2} \theta,
$$

with $\mathrm{d} \Omega=\sin \theta \mathrm{d} \theta \mathrm{d} \varphi$. To obtain the cross sections (Thomson, Rayleigh), we divide the intensity by the flux of the incoming plane wave, $\mathrm{d} \sigma^{\mathrm{T}, \mathrm{L}}=\mathrm{d} I^{\mathrm{T}, \mathrm{L}} /\langle|\boldsymbol{S}|\rangle$, which reads $\langle|\boldsymbol{S}|\rangle=2 c^{2} \tilde{\omega}^{-1} k(\tilde{\omega})|\hat{\boldsymbol{E}}|^{2}$ if $\hat{\boldsymbol{E}}$ is transversal, and $\langle|\boldsymbol{S}|\rangle=2 m_{\mathrm{t}}^{-2} \tilde{\omega} k(\tilde{\omega})|\hat{\boldsymbol{E}}|^{2}$ in the case of a longitudinally polarized $\hat{\boldsymbol{E}}$, cf (4.23)-(4.27). 
To exemplify the spectral densities (3.5), we replace in the oscillator equation (3.6) the monochromatic field by a pulse $\boldsymbol{E}=(2 \pi)^{-1} \int_{0}^{\infty} \boldsymbol{E}(\tilde{\omega}, t) \mathrm{d} \tilde{\omega}$. Substituting into (3.5) the Fourier-transformed oscillator velocity,

$$
\hat{\boldsymbol{v}}(\omega)=\frac{-\mathrm{i} \omega}{\omega_{0}^{2}-\omega^{2}-\mathrm{i} \gamma_{0} \omega} \frac{q_{0}}{m_{0}} \hat{\boldsymbol{E}}(\omega),
$$

we obtain the spectral densities of the radiated energy,

$\mathrm{d} E^{\mathrm{T}}=\frac{8}{3} \frac{q^{2} q_{0}^{2}}{(4 \pi)^{2}} \frac{|\hat{\boldsymbol{E}}(\omega)|^{2}}{m_{0}^{2} c^{2}} \frac{\omega^{3} k(\omega) \mathrm{d} \omega}{\left(\omega_{0}^{2}-\omega^{2}\right)^{2}+\left(\gamma_{0} \omega\right)^{2}}, \quad \mathrm{~d} E^{\mathrm{L}}=\frac{m_{\mathrm{t}}^{2} c^{2}}{2 \omega^{2}} \mathrm{~d} E^{\mathrm{T}}$.

Evaluating the integral of (3.13) at the resonance by steepest descent, we find for small linewidth the total transversally and longitudinally radiated energies $E^{\mathrm{T}, \mathrm{L}}=\int_{0}^{\infty} \mathrm{d} E^{\mathrm{T}, \mathrm{L}}(\omega)$ as

$$
E^{\mathrm{T}} \sim \frac{4 \pi}{3} \frac{q^{2} q_{0}^{2}}{(4 \pi)^{2}} \frac{k\left(\omega_{0}\right) \omega_{0}}{\gamma_{0} m_{0}^{2} c^{2}}\left|\hat{\boldsymbol{E}}\left(\omega_{0}\right)\right|^{2}, \quad \frac{E^{\mathrm{L}}}{E^{\mathrm{T}}} \sim \frac{m_{\mathrm{t}}^{2} c^{2}}{2 \omega_{0}^{2}} .
$$

In this oscillator example, we have used two different charges, $q$ and $q_{0}$. The space dependence of the field in (3.6) was neglected by appealing to a small oscillator size. Thus $\hat{\boldsymbol{E}}$ may be electric or tachyonic, and $q_{0}$ the electric or tachyonic charge of the oscillator. In the above formulae we considered tachyon radiation, so that $q$ and $k(\omega)$ are the tachyonic charge of the oscillator and the tachyonic wavevector of the emitted radiation. Comparing the spectral energy densities of the tachyonic and electromagnetic radiation (of an oscillator carrying both electric and tachyonic charge), we find

$$
\frac{\mathrm{d} E^{\mathrm{T}, \text { tach }}}{\mathrm{d} E^{\mathrm{em}}}=\frac{q^{2}}{e^{2}} \frac{c k(\omega)}{\omega}, \quad \frac{\mathrm{d} E^{\mathrm{L}, \text { tach }}}{\mathrm{d} E^{\mathrm{em}}}=\frac{1}{2} \frac{q^{2}}{e^{2}} m_{\mathrm{t}}^{2} c^{3} \frac{k(\omega)}{\omega^{3}} .
$$

A similar reasoning applies to the cross sections calculated above. It must be kept in mind, however, that these calculations are all done in dipole approximation, and spatial averaging effects will lower the right-hand sides of (3.15). In [14] we demonstrated that the maximal wavelength for tachyon radiation is about $1 \AA$, so that relation (3.15) can only be regarded as an order of magnitude estimate for atomic oscillators, the more so as it is classical. (We estimated the ratio of tachyonic and electric fine structure constants as $q^{2} / e^{2} \approx 1.4 \times 10^{-11}$, and the tachyon mass as $m_{\mathrm{t}} \approx m_{\mathrm{e}} / 238 \approx 2.15 \mathrm{keV} c^{-2}$.)

\section{Superluminal wave propagation in a permeable spacetime}

We will study tachyonic Proca fields in a spacetime with frequency-dependent permeabilities. The formalism is kept close to electrodynamics [29,30], which justifies brevity. Several parts of this section, in particular the discussion of energy, are based on [29]. The Fourier coefficients of the inductions and field strengths are supposed to relate in the usual way as

$$
\hat{\boldsymbol{D}}(\boldsymbol{x}, \omega)=\hat{\varepsilon}(\omega) \hat{\boldsymbol{E}}(\boldsymbol{x}, \omega), \quad \hat{\boldsymbol{B}}(\boldsymbol{x}, \omega)=\hat{\mu}(\omega) \hat{\boldsymbol{H}}(\boldsymbol{x}, \omega) .
$$

In real space, electric and magnetic inductions are denoted by $\boldsymbol{D}(\boldsymbol{x}, t)$ and $\boldsymbol{H}(\boldsymbol{x}, t)$, and the dielectric and magnetic permeabilities are $\varepsilon(t)$ and $\mu(t)$, respectively; $\kappa$ and $\chi$ denote the dielectric and magnetic susceptibilities, and $\boldsymbol{P}$ and $M$ are the polarization and magnetization vectors, so that in Fourier space $\hat{\boldsymbol{P}}=\hat{\kappa} \hat{\boldsymbol{E}}, \hat{\kappa}:=\hat{\varepsilon}-1$ and $\hat{\boldsymbol{M}}=\hat{\chi} \hat{\boldsymbol{H}}, \hat{\chi}:=\hat{\mu}-1$. We use the terminology and notation customary in electrodynamics; this is very suggestive and no confusion can arise, as we do not deal with electromagnetic fields. The tachyonic Lorentz force acting on a subluminal particle is composed of the field strengths $\boldsymbol{E}$ and $\boldsymbol{B}$, cf (1.3). All 
quantities in (4.1) are complex Fourier coefficients, their real-space expressions are defined as in (2.12) for the vector potential. The material relations (4.1) are not the most general ones; even if we stick to linearity, one may still assume $\hat{\boldsymbol{D}}_{m}=\hat{\varepsilon}_{m n} \hat{\boldsymbol{E}}_{n}$ in the case of anisotropy and inhomogeneity, and more importantly, $\hat{\boldsymbol{D}}=\hat{\varepsilon} \hat{\boldsymbol{E}}+\hat{\mu}_{0} \hat{\boldsymbol{B}}, \hat{\boldsymbol{B}}=\hat{\mu} \hat{\boldsymbol{H}}+\hat{\varepsilon}_{0} \hat{\boldsymbol{D}}$ is quite possible, as the condition $a / \lambda \ll 1$ (where $a$ is the size of the oscillators of the ether and $\lambda$ is the wavelength of the tachyon radiation) may not hold, cf the end of section 3 , but we do not consider these mild generalizations here, to save notation.

As in the massless case, the Maxwell equations in a permeable spacetime are obtained by replacing in the inhomogeneous equations in (2.4) the field strengths by inductions, $(\boldsymbol{E}, \boldsymbol{B}) \rightarrow(\boldsymbol{D}, \boldsymbol{H})$. Thus we find in Fourier space,

$$
\begin{array}{ll}
\operatorname{div} \hat{\boldsymbol{B}}=0, & \operatorname{rot} \hat{\boldsymbol{E}}-\mathrm{i} \omega c^{-1} \hat{\boldsymbol{B}}=0, \\
\operatorname{div} \hat{\boldsymbol{D}}=\hat{\rho}-c^{-1} m_{\mathrm{t}}^{2} \hat{a}_{0}, & \operatorname{rot} \hat{\boldsymbol{H}}+\mathrm{i} \omega c^{-1} \hat{\boldsymbol{D}}=c^{-1} \hat{\boldsymbol{j}}+m_{\mathrm{t}}^{2} \hat{\boldsymbol{a}}, \\
\hat{\boldsymbol{a}}(\omega) \hat{\mu}(\omega):=\hat{\boldsymbol{A}}(\omega), & \hat{a}_{0}(\omega):=\hat{\varepsilon}(\omega) \hat{A}_{0}(\omega) .
\end{array}
$$

The Lorentz condition reads $c^{2} \operatorname{div} \hat{\boldsymbol{a}}=-\mathrm{i} \omega \hat{a}_{0}$. All fields, but not the permeabilities, depend on the space coordinates as in (4.1).

In the following we will study wave propagation, and drop charge and current densities in (4.2). Applying the rotor to the rotor equations in (4.2), we find

$$
\left(\Delta+k^{2}(\omega)\right) \hat{\boldsymbol{E}}=0, \quad k(\omega):=\omega n / c, \quad n:=\sqrt{\hat{\varepsilon} \hat{\mu}+\left(m_{\mathrm{t}} c / \omega\right)^{2}},
$$

and the same for $\hat{\boldsymbol{B}}$ and the inductions. The refractive index $n(\omega)$ is in general complex, and we assume $\operatorname{Re} n(\omega)>0$; there is no wave motion if the real part is zero. To obtain a reasonable geometrical optics limit, it is crucial that all fields admit the same dispersion relation. This is achieved by the $\hat{\varepsilon}$ and $\hat{\mu}^{-1}$ scaling (4.3) of the vector potential; there is no other linear scaling possible to this effect. The material relations (4.3), complementing (4.1), are also strongly suggested by the positivity of energy, cf (4.17) and (4.19).

We consider $\hat{\boldsymbol{E}}(\boldsymbol{x}, \omega)=\boldsymbol{E}_{0}(\boldsymbol{x}, \omega) \exp (\mathrm{i} \psi(\boldsymbol{x}, \omega))$, with weakly varying $\boldsymbol{E}_{0}(\boldsymbol{x}, \omega)$, so that spatial derivatives can be neglected, and find from (4.4) the reduced eikonal equation $(\nabla \psi)^{2}=k^{2}(\omega)$. The eikonal is $\psi(\boldsymbol{x}, \omega)-\omega t$, and $\psi(\boldsymbol{x}, \omega)$ is assumed to be real, which means $k^{2}(\omega)>0$. (Absorption is beyond the geometrical optics limit.) The wavevector is thus $k=\nabla \psi=k_{0} c^{-1} \omega n(\omega)$, with a real unit vector $\boldsymbol{k}_{0}$ and a positive refractive index. The phase and group velocity read as $v_{\mathrm{ph}}=\omega / k$ and

$$
\frac{1}{v_{\mathrm{gr}}}=\frac{\mathrm{d} k}{\mathrm{~d} \omega}=\frac{1}{2 c n}\left(\frac{\mathrm{d}(\omega \hat{\varepsilon})}{\mathrm{d} \omega} \hat{\mu}+\frac{\mathrm{d}(\omega \hat{\mu})}{\mathrm{d} \omega} \hat{\varepsilon}\right),
$$

the latter reduces in the case of frequency-independent permeabilities to $\mathrm{d} \omega / \mathrm{d} k=c n /(\hat{\varepsilon} \hat{\mu})$.

Relations (4.1) amount to the usual convolutions in real space,

$$
\begin{aligned}
& \boldsymbol{D}(t)=\boldsymbol{E}(t)+\int_{-\infty}^{+\infty} \kappa\left(t^{\prime}\right) \boldsymbol{E}\left(t-t^{\prime}\right) \mathrm{d} t^{\prime}, \\
& \boldsymbol{B}(t)=\boldsymbol{H}(t)+\int_{-\infty}^{+\infty} \chi\left(t^{\prime}\right) \boldsymbol{H}\left(t-t^{\prime}\right) \mathrm{d} t^{\prime},
\end{aligned}
$$

so that $\varepsilon(t)=\delta(t)+\kappa(t), \mu(t)=\delta(t)+\chi(t)$, with the Dirac $\delta$-function. In real space, the Maxwell equations have likewise a very familiar form,

$$
\begin{array}{rlrl}
\operatorname{div} \boldsymbol{B} & =0, & & \operatorname{rot} \boldsymbol{E}+c^{-1} \partial \boldsymbol{B} / \partial t=0, \\
\operatorname{div} \boldsymbol{D}=\rho-c^{-1} m_{\mathrm{t}}^{2} a_{0}, & & \operatorname{rot} \boldsymbol{H}-c^{-1} \partial \boldsymbol{D} / \partial t=c^{-1} \boldsymbol{j}+m_{\mathrm{t}}^{2} \boldsymbol{a},
\end{array}
$$


with the Lorentz condition $\partial a_{0} / \partial t=c^{2} \operatorname{div} \boldsymbol{a}$. The material equations (4.3) are equivalent to

$$
\begin{aligned}
& a_{0}(t)=A_{0}(t)+\int_{-\infty}^{+\infty} \kappa\left(t^{\prime}\right) A_{0}\left(t-t^{\prime}\right) \mathrm{d} t^{\prime} \\
& \boldsymbol{A}(t)=\boldsymbol{a}(t)+\int_{-\infty}^{+\infty} \chi\left(t^{\prime}\right) \boldsymbol{a}\left(t-t^{\prime}\right) \mathrm{d} t^{\prime}
\end{aligned}
$$

These integral equations as well as (4.6) can be iteratively inverted by a standard procedure. The response of the medium cannot happen prior to its exposure to the field, which means that $\kappa(t)$ and $\chi(t)$ are required to vanish identically for negative $t$. Thus the lower integration boundary in (4.6) and (4.8) can be replaced by zero, provided there are no distributional singularities at $t=0$ in the integrands. It should be stressed that all equations in this section are non-covariant, as the permeabilities $\varepsilon(t)$ and $\mu(t)$ are defined in a special reference frame, the rest frame of the ether. The ether defines an absolute space, and therefore relativistic invariance is not required, cf section 1.

We turn to energy and define the Poynting vector as $\boldsymbol{S}=c \boldsymbol{E} \times \boldsymbol{H}+m_{\mathrm{t}}^{2} A_{0} \boldsymbol{a}, \operatorname{cf}(2.6)$. The Maxwell equations (4.7) and the material relations (4.1) and (4.3) result in

$$
\operatorname{div} \boldsymbol{S}+\boldsymbol{E} \cdot \frac{\partial \boldsymbol{D}}{\partial t}+\boldsymbol{H} \cdot \frac{\partial \boldsymbol{B}}{\partial t}-m_{\mathrm{t}}^{2}\left(\frac{1}{c^{2}} A_{0} \frac{\partial a_{0}}{\partial t}+\boldsymbol{a} \cdot \frac{\partial \boldsymbol{A}}{\partial t}\right)=-\boldsymbol{j} \cdot \boldsymbol{E} .
$$

If $\varepsilon$ and $\mu$ are constant and $j=0$, we may write, $\operatorname{cf}(2.5)$ and (2.6),

$$
\frac{\partial \rho_{E}}{\partial t}+\operatorname{div} \boldsymbol{S}=0, \quad \rho_{E}=\frac{1}{2}(\boldsymbol{E} \cdot \boldsymbol{D}+\boldsymbol{H} \cdot \boldsymbol{B})-\frac{1}{2} m_{\mathrm{t}}^{2}\left(c^{-2} A_{0} a_{0}+\boldsymbol{a} \cdot \boldsymbol{A}\right) .
$$

A positive-definite energy density can be extracted from (4.9) by time averaging. To this end, we consider frequency-dependent permeabilities and a wave train $\boldsymbol{E}(t, \boldsymbol{x})=\tilde{\boldsymbol{E}}+\tilde{\boldsymbol{E}}^{*}$, $\tilde{\boldsymbol{E}}:=\boldsymbol{E}_{0}(t, \boldsymbol{x}) \mathrm{e}^{-\mathrm{i} \omega_{0} t}$, where $\boldsymbol{E}_{0}$ is a slowly varying function of time as compared with the variation of the exponential, so that the average of $\boldsymbol{E}^{2}$ over a period of $2 \pi / \omega_{0}$ is $\left\langle\boldsymbol{E}^{2}\right\rangle=2 \boldsymbol{E}_{0} \cdot \boldsymbol{E}_{0}^{*}$. Defining $\boldsymbol{D}=\tilde{\boldsymbol{D}}+\tilde{\boldsymbol{D}}^{*}$, we find

$$
\frac{\partial \tilde{\boldsymbol{D}}}{\partial t}=\frac{-\mathrm{i}}{2 \pi} \int_{-\infty}^{+\infty} \omega \hat{\varepsilon}(\omega) \hat{\boldsymbol{E}}(\omega) \mathrm{e}^{-\mathrm{i} \omega t} \mathrm{~d} \omega, \quad \hat{\boldsymbol{E}}(\omega)=\int_{-\infty}^{+\infty} \boldsymbol{E}_{0}(t, \boldsymbol{x}) \mathrm{e}^{\mathrm{i}\left(\omega-\omega_{0}\right) t} \mathrm{~d} t
$$

By expanding $\omega \hat{\varepsilon}(\omega)$ around $\omega_{0}$ and interchanging integrations, we obtain

$$
\frac{\partial \tilde{\boldsymbol{D}}}{\partial t}=\mathrm{e}^{-\mathrm{i} \omega_{0} t}\left(-\mathrm{i} \omega_{0} \hat{\varepsilon}\left(\omega_{0}\right) \boldsymbol{E}_{0}(t, \boldsymbol{x})+\frac{\partial \boldsymbol{E}_{0}}{\partial t} \frac{\mathrm{d}\left(\omega_{0} \hat{\varepsilon}\left(\omega_{0}\right)\right)}{\mathrm{d} \omega_{0}}+\cdots\right)
$$

In (4.11) and (4.12) we may replace $(\boldsymbol{E}, \boldsymbol{D}, \hat{\varepsilon})$ by $(\boldsymbol{H}, \boldsymbol{B}, \hat{\mu})$, as well as by $\left(A_{0}, a_{0}, \hat{\varepsilon}\right)$ and $(\boldsymbol{a}, \boldsymbol{A}, \hat{\mu})$. The time averaging of (4.9) is effected by means of

$$
\left\langle\boldsymbol{E} \cdot \frac{\partial \boldsymbol{D}}{\partial t}\right\rangle=\tilde{\boldsymbol{E}}^{*} \cdot \frac{\partial \tilde{\boldsymbol{D}}}{\partial t}+\tilde{\boldsymbol{E}} \cdot \frac{\partial \tilde{\boldsymbol{D}}^{*}}{\partial t} \sim 2 \omega_{0} \operatorname{Im} \hat{\varepsilon}\left(\omega_{0}\right)|\tilde{\boldsymbol{E}}|^{2}+\operatorname{Re} \frac{\mathrm{d}\left(\omega_{0} \hat{\varepsilon}\left(\omega_{0}\right)\right)}{\mathrm{d} \omega_{0}} \frac{\partial}{\partial t}|\tilde{\boldsymbol{E}}|^{2},
$$

and by identical equations for $(\boldsymbol{H}, \boldsymbol{B}, \hat{\mu})$, etc. Identifying the averaged energy density and the Poynting vector as

$$
\begin{aligned}
& \left\langle\rho_{E}\right\rangle \sim \operatorname{Re} \frac{\mathrm{d}(\omega \hat{\varepsilon})}{\mathrm{d} \omega}\left(\tilde{\boldsymbol{E}} \cdot \tilde{\boldsymbol{E}}^{*}-m_{\mathrm{t}}^{2} c^{-2} \tilde{A}_{0} \tilde{A}_{0}^{*}\right)+\operatorname{Re} \frac{\mathrm{d}(\omega \hat{\mu})}{\mathrm{d} \omega}\left(\tilde{\boldsymbol{H}} \cdot \tilde{\boldsymbol{H}}^{*}-m_{\mathrm{t}}^{2} \tilde{\boldsymbol{a}} \cdot \tilde{\boldsymbol{a}}^{*}\right), \\
& \langle\boldsymbol{S}\rangle \sim c \tilde{\boldsymbol{E}} \times \tilde{\boldsymbol{H}}^{*}+c \tilde{\boldsymbol{E}}^{*} \times \tilde{\boldsymbol{H}}+m_{\mathrm{t}}^{2}\left(\tilde{A}_{0}^{*} \tilde{\boldsymbol{a}}+\tilde{A}_{0} \tilde{\boldsymbol{a}}^{*}\right),
\end{aligned}
$$


we can write the averaged conservation law (4.9) $(j=0)$ as

$-\operatorname{div}\langle\boldsymbol{S}\rangle \sim \frac{\partial\left\langle\rho_{E}\right\rangle}{\partial t}+2 \omega_{0}\left(\left(\tilde{\boldsymbol{E}} \cdot \tilde{\boldsymbol{E}}^{*}-m_{\mathrm{t}}^{2} c^{-2} \tilde{A}_{0} \tilde{A}_{0}^{*}\right) \operatorname{Im} \hat{\varepsilon}+\left(\tilde{\boldsymbol{H}} \cdot \tilde{\boldsymbol{H}}^{*}-m_{\mathrm{t}}^{2} \tilde{\boldsymbol{a}} \cdot \tilde{\boldsymbol{a}}^{*}\right) \operatorname{Im} \hat{\mu}\right)$.

In (4.14)-(4.16) we may substitute $\tilde{\boldsymbol{E}} \cdot \tilde{\boldsymbol{E}}^{*}=\frac{1}{2}\left\langle\boldsymbol{E}^{2}\right\rangle, \tilde{\boldsymbol{E}} \times \tilde{\boldsymbol{H}}^{*}+\tilde{\boldsymbol{E}}^{*} \times \tilde{\boldsymbol{H}}=\langle\boldsymbol{E} \times \boldsymbol{H}\rangle$, etc. The density (4.14) is positive for transversal modes and negative for longitudinal ones, which suggests to define the flux vector as well as the energy density for longitudinal modes with opposite sign. We so find, analogously to (2.19) and (2.20),

$$
\begin{aligned}
&\left\langle\rho_{\mathrm{E}}^{\mathrm{T}}\right\rangle \sim \frac{1}{2} \operatorname{Re} \frac{\mathrm{d}(\omega \hat{\varepsilon})}{\mathrm{d} \omega}\left\langle\boldsymbol{E}^{\mathrm{T} 2}\right\rangle+\frac{1}{2} \operatorname{Re} \frac{\mathrm{d}(\omega \hat{\mu})}{\mathrm{d} \omega}\left(\left\langle\boldsymbol{H}^{\mathrm{T} 2}\right\rangle-m_{\mathrm{t}}^{2}\left\langle\boldsymbol{a}^{\mathrm{T} 2}\right\rangle\right), \\
&\left\langle\rho_{\mathrm{E}}^{\mathrm{L}}\right\rangle \sim \frac{m_{\mathrm{t}}^{2}}{2} \operatorname{Re} \frac{\mathrm{d}(\omega \hat{\mu})}{\mathrm{d} \omega}\left\langle\boldsymbol{a}^{\mathrm{L} 2}\right\rangle+\frac{1}{2} \operatorname{Re} \frac{\mathrm{d}(\omega \hat{\varepsilon})}{\mathrm{d} \omega}\left(m_{\mathrm{t}}^{2} c^{-2}\left\langle A_{0}^{\mathrm{L} 2}\right\rangle-\left\langle\boldsymbol{E}^{\mathrm{L} 2}\right\rangle\right), \\
&\left\langle\boldsymbol{S}^{\mathrm{T}}\right\rangle \sim c\left\langle\boldsymbol{E}^{\mathrm{T}} \times \boldsymbol{H}^{\mathrm{T}}\right\rangle, \quad\left\langle\boldsymbol{S}^{\mathrm{L}}\right\rangle \sim-m_{\mathrm{t}}^{2}\left\langle A_{0}^{\mathrm{L}} \boldsymbol{a}^{\mathrm{L}}\right\rangle .
\end{aligned}
$$

The energy balance thus reads

$$
\begin{aligned}
& \partial\left\langle\rho_{\mathrm{E}}^{\mathrm{T}, \mathrm{L}}\right\rangle / \partial t+\operatorname{div}\left\langle\boldsymbol{S}^{\mathrm{T}, \mathrm{L}}\right\rangle=-\left\langle I_{\mathrm{dis}}^{\mathrm{T}, \mathrm{L}}\right\rangle, \\
& \left\langle I_{\mathrm{dis}}^{\mathrm{T}}\right\rangle \sim \omega \operatorname{Im} \hat{\varepsilon}(\omega)\left\langle\boldsymbol{E}^{\mathrm{T} 2}\right\rangle+\omega \operatorname{Im} \hat{\mu}(\omega)\left(\left\langle\boldsymbol{H}^{\mathrm{T} 2}\right\rangle-m_{\mathrm{t}}^{2}\left\langle\boldsymbol{a}^{\mathrm{T} 2}\right\rangle\right), \\
& \left\langle I_{\mathrm{dis}}^{\mathrm{L}}\right\rangle \sim \omega \operatorname{Im} \hat{\mu}(\omega) m_{\mathrm{t}}^{2}\left\langle\boldsymbol{a}^{\mathrm{L} 2}\right\rangle+\omega \operatorname{Im} \hat{\varepsilon}(\omega)\left(m_{\mathrm{t}}^{2} c^{-2}\left\langle A_{0}^{\mathrm{L} 2}\right\rangle-\left\langle\boldsymbol{E}^{\mathrm{L} 2}\right\rangle\right),
\end{aligned}
$$

where $\left\langle I_{\text {dis }}^{\mathrm{T}, \mathrm{L}}\right\rangle$ is the energy per unit time dissipated into the ether. This energy is positive, which requires the positivity of the imaginary components of the permeabilities. The real parts of the derivatives in (4.17) have to be positive for the same reason.

We apply this energy concept to damped plane waves, $\hat{\boldsymbol{A}}(\boldsymbol{x}, \omega)=\tilde{\boldsymbol{A}}(\omega) \mathrm{e}^{\mathrm{i} k \cdot \boldsymbol{x}}, \hat{\boldsymbol{E}}(\boldsymbol{x}, \omega)=$ $\tilde{\boldsymbol{E}}(\omega) \mathrm{e}^{\mathrm{i} \boldsymbol{k} \cdot \boldsymbol{x}}$, etc, where $\boldsymbol{k}:=\boldsymbol{k}_{0} c^{-1} \omega n(\omega)$, with a real unit vector $\boldsymbol{k}_{0}$ and a complex refractive index $n(\omega)$ defined in (4.4). As above, the real fields are $\boldsymbol{E}(\boldsymbol{x}, t)=\hat{\boldsymbol{E}} \mathrm{e}^{-\mathrm{i} \omega t}+\hat{\boldsymbol{E}}^{*} \mathrm{e}^{\mathrm{i} \omega t}$, etc. As $\operatorname{Re} n(\omega)>0$, the retarded propagation is in the direction of $\boldsymbol{k}_{0}$, also for negative $\omega$. In the Maxwell equations (4.2) we put current and charge density to zero, so that

$$
\begin{array}{ll}
\boldsymbol{k} \cdot \hat{\boldsymbol{B}}=0, & \boldsymbol{k} \times \hat{\boldsymbol{E}}-\omega c^{-1} \hat{\boldsymbol{B}}=0, \\
\boldsymbol{k} \cdot \hat{\boldsymbol{E}}=\mathrm{i} c^{-1} m_{\mathrm{t}}^{2} \hat{A}_{0}, & \boldsymbol{k} \times \hat{\boldsymbol{B}}+\omega c^{-1} \hat{\varepsilon}(\omega) \hat{\mu}(\omega) \hat{\boldsymbol{E}}=-\mathrm{i} m_{\mathrm{t}}^{2} \hat{\boldsymbol{A}},
\end{array}
$$

with the Lorentz condition $\omega c^{-2} \hat{a}_{0}+\boldsymbol{k} \cdot \hat{\boldsymbol{a}}=0$. Clearly, $\boldsymbol{k}^{2}=k^{2}(\omega)$, cf (4.4), which is in general complex. In (4.4) we write $n=: n_{\mathrm{Re}}+\mathrm{i} n_{\mathrm{Im}}, n_{\mathrm{Re}}>0$,

$$
\begin{array}{ll}
n_{\mathrm{Re}}=\frac{1}{\sqrt{2}} \sqrt{\sqrt{\alpha^{2}+\beta^{2}}+\alpha}, & n_{\mathrm{Im}}=\frac{\operatorname{sign}(\beta)}{\sqrt{2}} \sqrt{\sqrt{\alpha^{2}+\beta^{2}}-\alpha}, \\
\alpha:=\operatorname{Re}(\hat{\varepsilon} \hat{\mu})+\left(m_{\mathrm{t}} c / \omega\right)^{2}, & \beta:=\operatorname{Im}(\hat{\varepsilon} \hat{\mu}) .
\end{array}
$$

A retarded damped (rather than amplified) wave requires a positive absorptive coefficient $n_{\operatorname{Im}}$, so that $\operatorname{Im}(\hat{\varepsilon} \hat{\mu}) \geqslant 0$, a third positivity condition on the permeabilities, see after (4.19).

Transversality means

$$
\hat{\boldsymbol{A}}^{\mathrm{T}}(\omega) \cdot \boldsymbol{k}=0, \quad \hat{A}_{0}^{\mathrm{T}}=0, \quad \hat{\boldsymbol{E}}^{\mathrm{T}}=\mathrm{i}(\omega / c) \hat{\boldsymbol{A}}^{\mathrm{T}}, \quad \hat{\boldsymbol{B}}^{\mathrm{T}}=\mathrm{i} \boldsymbol{k} \times \hat{\boldsymbol{A}}^{\mathrm{T}},
$$

and the transversal energy density and flux are readily obtained as

$$
\begin{aligned}
& \left\langle\rho_{\mathrm{E}}^{\mathrm{T}}\right\rangle=\frac{\omega^{2}}{c^{2}}\left|\hat{\boldsymbol{A}}^{\mathrm{T}}\right|^{2}\left(\operatorname{Re} \frac{\mathrm{d}(\omega \hat{\varepsilon})}{\mathrm{d} \omega}+\operatorname{Re} \frac{\mathrm{d}(\omega \hat{\mu})}{\mathrm{d} \omega} \frac{1}{|\hat{\mu}|^{2}}\left(|n|^{2}-\frac{m_{\mathrm{t}}^{2} c^{2}}{\omega^{2}}\right)\right), \\
& \left\langle\boldsymbol{S}^{\mathrm{T}}\right\rangle=2 \frac{\omega^{2}}{c}\left|\hat{\boldsymbol{A}}^{\mathrm{T}}\right|^{2} \operatorname{Re}(n / \hat{\mu}) \boldsymbol{k}_{0},
\end{aligned}
$$


with $|n|^{2}=\sqrt{\alpha^{2}+\beta^{2}}$, cf (4.22). In a similar manner, for longitudinal modes,

$|\boldsymbol{k}|^{2} \hat{\boldsymbol{A}}^{\mathrm{L}}=\left(\hat{\boldsymbol{A}}^{\mathrm{L}} \cdot \boldsymbol{k}\right) \boldsymbol{k}, \quad \hat{A}_{0}^{\mathrm{L}}=-\frac{c^{2}}{\hat{\varepsilon} \hat{\mu} \omega}\left(\hat{\boldsymbol{A}}^{\mathrm{L}} \cdot \boldsymbol{k}\right), \quad \hat{\boldsymbol{E}}^{\mathrm{L}}=-\mathrm{i} \frac{m_{\mathrm{t}}^{2} c}{\hat{\varepsilon} \hat{\mu} \omega} \hat{\boldsymbol{A}}^{\mathrm{L}}$,

and $\hat{\boldsymbol{B}}^{\mathrm{L}}=0$, so that the energy and the flux read

$$
\begin{aligned}
& \left\langle\rho_{\mathrm{E}}^{\mathrm{L}}\right\rangle=m_{\mathrm{t}}^{2}\left|\hat{\boldsymbol{a}}^{\mathrm{L}}\right|^{2}\left(\operatorname{Re} \frac{\mathrm{d}(\omega \hat{\varepsilon})}{\mathrm{d} \omega} \frac{1}{|\hat{\varepsilon}|^{2}}\left(|n|^{2}-\frac{m_{\mathrm{t}}^{2} c^{2}}{\omega^{2}}\right)+\operatorname{Re} \frac{\mathrm{d}(\omega \hat{\mu})}{\mathrm{d} \omega}\right), \\
& \left\langle\boldsymbol{S}^{\mathrm{L}}\right\rangle=2 m_{\mathrm{t}}^{2} c \operatorname{Re}(n / \hat{\varepsilon})\left|\hat{\boldsymbol{a}}^{\mathrm{L}}\right|^{2} \boldsymbol{k}_{0} .
\end{aligned}
$$

In (4.24) and (4.26) we may finally substitute the averaged wave fields

$$
\left|\hat{\boldsymbol{A}}^{\mathrm{T}}\right|^{2}=\frac{1}{2}\left\langle\boldsymbol{A}^{\mathrm{T} 2}\right\rangle=\frac{1}{2} \frac{c^{2}}{\omega^{2}}\left\langle\boldsymbol{E}^{\mathrm{T} 2}\right\rangle, \quad\left|\hat{\boldsymbol{a}}^{\mathrm{L}}\right|^{2}=\frac{1}{2}\left\langle\boldsymbol{a}^{\mathrm{L} 2}\right\rangle=\frac{1}{2} \frac{\omega^{2}}{m_{\mathrm{t}}^{4} c^{2}}\left\langle\boldsymbol{D}^{\mathrm{L} 2}\right\rangle .
$$

In the absence of damping, for real permeabilities, we obtain the familiar identity $\left\langle\boldsymbol{S}^{\mathrm{T}, \mathrm{L}}\right\rangle=$ $\boldsymbol{k}_{0}\left\langle\rho_{\mathrm{E}}^{\mathrm{T}, \mathrm{L}}\right\rangle \mathrm{d} \omega / \mathrm{d} k$, which just means that the energy propagates with group velocity.

As a first guess on the microscopic structure of the ether, we consider a classical oscillator model, which gives $\hat{\mu}=1$ and

$$
\begin{aligned}
& \hat{\varepsilon}(\omega)=1+\frac{N_{0} q_{0}^{2}}{m_{0}} g(\omega)=1-\frac{N_{0} q_{0}^{2}}{m_{0}} \frac{1}{\omega^{2}}\left(1-\frac{\mathrm{i} \gamma_{0}}{\omega}+\cdots\right), \\
& g(\omega):=\left(\omega_{0}^{2}-\omega^{2}-\mathrm{i} \gamma_{0} \omega\right)^{-1}, \quad \gamma_{0}>0 .
\end{aligned}
$$

As in (3.6), $\omega_{0}$ is the oscillator frequency, $\gamma_{0}$ the damping constant, $m_{0}$ the mass and $q_{0}$ the tachyonic charge of the uniformly distributed oscillators constituting the ether. The Drude formula (4.28) follows from (3.6), as $N_{0}$ dipoles $q_{0} \hat{\boldsymbol{r}}$ per unit volume generate the polarization $\hat{\boldsymbol{P}} \mathrm{e}^{-\mathrm{i} \omega t}=N_{0} q_{0} \hat{\boldsymbol{r}}=(\hat{\varepsilon}-1) \hat{\boldsymbol{E}} \mathrm{e}^{-\mathrm{i} \omega t}$. If $\omega_{0}=0$, we write $\hat{\varepsilon}=1+\mathrm{i} \sigma(\omega) / \omega$, with the conductivity $\sigma(\omega):=\left(N_{0} q_{0}^{2} / m_{0}\right)\left(\gamma_{0}-\mathrm{i} \omega\right)^{-1}$, so that $\hat{\varepsilon} \sim \mathrm{i} \sigma(0) / \omega$, for $\omega \rightarrow 0$, and thus $n_{\mathrm{Re}} \sim m_{\mathrm{t}} c / \omega$ and $n_{\mathrm{Im}} \sim \sigma(0) /\left(2 m_{\mathrm{t}} c\right)$, which is very different from the massless case, $n_{\mathrm{Re}} \sim n_{\mathrm{Im}} \sim \sqrt{\sigma(0) /(2 \omega)}$. For finite $\omega_{0}$, the dielectric susceptibility $\kappa(t)$, cf (4.6), is readily obtained by Fourier transforming $\hat{\varepsilon}(\omega)-1$,

$$
\kappa(t)=\frac{N_{0} q_{0}^{2}}{2 \pi m_{0}} \int_{-\infty}^{+\infty} g(\omega) \mathrm{e}^{-\mathrm{i} \omega t} \mathrm{~d} \omega=\frac{N_{0} q_{0}^{2}}{m_{0}} \frac{\mathrm{e}^{-\gamma_{0} t / 2}}{2 \mathrm{i} \omega_{0}}\left(\mathrm{e}^{\mathrm{i} \omega_{0} t}-\mathrm{e}^{-\mathrm{i} \omega_{0} t}\right) \theta(t) .
$$

A further term $\mathrm{i} \gamma_{(3)} \omega^{3}$ is sometimes added to the denominators in (3.6) and $g(\omega)$ in (4.29), corresponding to a self-interaction (Abraham-Lorentz) $\gamma_{(3)} \hat{\boldsymbol{r}}^{\prime \prime \prime}$ on the left-hand side of the oscillator equation in (3.6), ef [30-32], but this classical radiation damping is known to be causality violating due to pre-acceleration. We will return to (4.28) in the next section, when we discuss the absorber theory.

\section{Time-symmetric wave propagation in the ether}

The wave propagation discussed in sections 2 and 3 also applies to the permeable, absorbing spacetime introduced in section 4 . The only change needed is to replace in the Fourier components of the fields the positive $k(\omega)$ defined in (2.9) by $\omega n(\omega) / c$ in (4.4). This holds, in particular, for the modes (2.14)-(2.18), the Green function (2.10), the current (3.3) and the vector potential in (3.4). In the first part of this section we sketch, using a heuristic argument taken over from [26] with some minor modifications, how advanced modes generated by 
the time-symmetric Green function (2.10) (defined with $k(\omega)$ in (4.4)) can be converted into retarded ones by the oscillators of the ether. In (5.13)-(5.18) we will show how retarded waves can be generated by a subluminal current, without reference to the microscopic structure of the ether. This is less intuitive, but it demonstrates quite explicitly causal superluminal wave propagation in the ether without approximations. We will give the heuristic arguments for transversal waves only; the formal derivation starting from (5.12) is valid for longitudinal modes as well.

According to (3.4) and (2.17), the transversal retarded field of the subluminal source is given by

$$
\hat{\boldsymbol{E}}^{\mathrm{ret}}(\boldsymbol{x}, \omega) \sim \frac{q}{4 \pi c^{2}} \frac{\mathrm{i} \omega}{r} \exp \left(\mathrm{i} \frac{n(\omega) \omega}{c} r\right)(\hat{\boldsymbol{v}}(\omega)-(\boldsymbol{e} \cdot \hat{\boldsymbol{v}}(\omega)) \boldsymbol{e}),
$$

with $e:=\boldsymbol{x} / r$ and $n(\omega)=\sqrt{\hat{\varepsilon} \hat{\mu}+\left(m_{\mathrm{t}} c / \omega\right)^{2}}$ as in (4.4). We assume $\hat{\boldsymbol{v}}(\omega)$ as real; elliptically polarized modes can be handled by superposition. This field acts on the oscillators of the ether as in (3.6). The permeabilities entering in $n(\omega)$ are $\hat{\mu}=1$ and $\hat{\varepsilon}(\omega)$ defined in (4.28), so that

$$
n(\omega)-n_{0}(\omega)=\frac{1}{2} N_{0} \frac{q_{0}^{2}}{m_{0}} \frac{g(\omega)}{n_{0}(\omega)}+\mathrm{O}\left(N_{0}^{2} q_{0}^{4} / m_{0}^{2}\right),
$$

where $n_{0}(\omega)$ is defined with $\hat{\varepsilon}=\hat{\mu}=1$, see after (2.14), $N_{0}$ is the number density, $q_{0}$ and $m_{0}$ are the tachyonic charge and the mass of the subluminal oscillators, and $g(\omega)$ is defined in (4.29). The velocity of an oscillator at $\boldsymbol{x}_{k}$, driven by the retarded field (5.1), is according to (3.12),

$$
\hat{\boldsymbol{v}}_{k}(\omega)=-\mathrm{i} \omega g(\omega)\left(q_{0} / m_{0}\right) \hat{\boldsymbol{E}}^{\mathrm{ret}}\left(\boldsymbol{x}_{k}, \omega\right)
$$

The absorber theory does not really depend on the numerical values of the constants in (5.2), even not on the shape of $g(\omega)$ as long as we assume relation (5.3). It will become evident in the subsequent derivation that the electric permeability merely serves as a regularizer of an otherwise divergent integral. To that end a positive imaginary component of $g(\omega)$ is required, that is, a positive absorptive coefficient resulting in exponential decay of the retarded wave fields. To get rid of the exponential damping in the final result, we regard $N_{0} q_{0}^{2} g(\omega) / m_{0}$ in (5.2) as $\varepsilon$-small, with an arbitrary real and positive imaginary part, so that (5.1) is only infinitesimally damped.

The retarded field (5.1) is assumed to be composed of a time-symmetric initial field and an absorber field, both acausal and thus non-observable, as they contain advanced components which, however, cancel if added. To assemble the absorber field, we start with the advanced component of the tachyon field generated by the oscillator $\left(\boldsymbol{x}_{k}, \hat{\boldsymbol{v}}_{k}\right)$, cf (2.15) and (5.1), that is

$\hat{\boldsymbol{E}}_{k}^{\mathrm{adv}}(\boldsymbol{x}, \omega) \sim \frac{q_{0}}{8 \pi c^{2}} \frac{\mathrm{i} \omega}{\left|\boldsymbol{x}-\boldsymbol{x}_{k}\right|} \exp \left(-\mathrm{i} \frac{\omega}{c} n_{0}(\omega)\left|\boldsymbol{x}-\boldsymbol{x}_{k}\right|\right)\left(\hat{\boldsymbol{v}}_{k}-\left(\tilde{\boldsymbol{e}}_{k} \cdot \hat{\boldsymbol{v}}_{k}\right) \tilde{\boldsymbol{e}}_{k}\right)$,

with $\tilde{\boldsymbol{e}}_{k}:=\left(\boldsymbol{x}-\boldsymbol{x}_{k}\right) /\left|\boldsymbol{x}-\boldsymbol{x}_{k}\right|$. (Advanced components of the oscillator fields are not affected by the permeability generated by the oscillators themselves.) We put $\left|\boldsymbol{x}-\boldsymbol{x}_{k}\right|=$ $r_{k}-r \cos \left(\boldsymbol{x}, \boldsymbol{x}_{k}\right)+\mathrm{O}\left(1 / r_{k}\right), \cos \left(\boldsymbol{x}, \boldsymbol{x}_{k}\right):=\boldsymbol{x}^{0} \cdot \boldsymbol{x}_{k}^{0}$ (real unit vectors are denoted by a zero superscript in this section), as well as $\tilde{e}_{k} \approx-e_{k}$. Hence, via (5.1) and (5.3),

$$
\begin{aligned}
\hat{\boldsymbol{E}}_{k}^{\mathrm{adv}}(\boldsymbol{x}, \omega) \sim & \frac{\mathrm{i}}{2\left(4 \pi c^{2}\right)^{2}} \frac{q q_{0}^{2}}{m_{0}} \frac{\omega^{3} g(\omega)}{r_{k}^{2}} \exp \left(\mathrm{i} c^{-1} \omega\left(n(\omega)-n_{0}(\omega)\right) r_{k}\right) \\
& \times \exp \left(\mathrm{i} c^{-1} \omega n_{0}(\omega) r \cos \left(\boldsymbol{x}, \boldsymbol{x}_{k}\right)\right)\left(\hat{\boldsymbol{v}}-\left(\boldsymbol{e}_{k} \cdot \hat{\boldsymbol{v}}\right) \boldsymbol{e}_{k}\right) .
\end{aligned}
$$


We consider the projection of $\hat{\boldsymbol{E}}_{k}^{\mathrm{adv}}(\boldsymbol{x}, \omega)$ onto the velocity, $\left(\hat{\boldsymbol{E}}_{k}^{\mathrm{adv}} \cdot \hat{\boldsymbol{v}}^{0}\right) \hat{\boldsymbol{v}}^{0}$, which amounts to replacing in (5.5) $\left(\hat{\boldsymbol{v}}-\left(\boldsymbol{e}_{k} \cdot \hat{\boldsymbol{v}}\right) \boldsymbol{e}_{k}\right)$ by $\hat{\boldsymbol{v}} \sin ^{2}\left(\boldsymbol{e}_{k} \cdot \hat{\boldsymbol{v}}^{0}\right)$ and we sum over all oscillators, so that the total advanced response generated by the absorber is given by

$$
\left(\hat{\boldsymbol{E}}^{\mathrm{abs}}(\boldsymbol{x}, \omega) \cdot \hat{\boldsymbol{v}}^{0}\right) \hat{\boldsymbol{v}}^{0} \sim N_{0} \hat{\boldsymbol{v}}^{0} \int\left(\hat{\boldsymbol{E}}_{k}^{\mathrm{adv}} \cdot \hat{\boldsymbol{v}}^{0}\right) r_{k}^{2} \mathrm{~d} r_{k} \mathrm{~d} \Omega\left(\boldsymbol{x}_{k}^{0}\right)
$$

integrated over the whole 3 -space. The radial integral is trivial, and we obtain

$$
\begin{aligned}
& \left(\hat{\boldsymbol{E}}^{\mathrm{abs}} \cdot \hat{\boldsymbol{v}}^{0}\right) \hat{\boldsymbol{v}}^{0} \sim \frac{-q \omega^{2}}{(4 \pi)^{2} c^{3}} \frac{N_{0} q_{0}^{2}}{m_{0}} \frac{g(\omega)}{2\left(n(\omega)-n_{0}(\omega)\right)} A\left(\omega, \boldsymbol{x}, \hat{\boldsymbol{v}}^{0}\right) \hat{\boldsymbol{v}}(\omega), \\
& A\left(\omega, \boldsymbol{x}, \hat{\boldsymbol{v}}^{0}\right):=\int \mathrm{d} \Omega\left(\boldsymbol{x}_{k}^{0}\right) \exp \left(\mathrm{i} \frac{\omega n_{0}(\omega)}{c} r \cos \left(\boldsymbol{x}, \boldsymbol{x}_{k}\right)\right) \sin ^{2}\left(\hat{\boldsymbol{v}}^{0}, \boldsymbol{x}_{k}\right) .
\end{aligned}
$$

The solid angle element $\mathrm{d} \Omega=\sin \theta \mathrm{d} \theta \mathrm{d} \varphi$ refers to the polar coordinates of $\boldsymbol{x}_{k}=\left(r_{k}, \theta, \varphi\right)$, the polar axis is chosen in the direction of $\boldsymbol{x}$. We write $\theta=\left(\boldsymbol{x}, \boldsymbol{x}_{k}\right)$ and $\chi:=\left(\hat{\boldsymbol{v}}^{0}, \boldsymbol{x}_{k}\right)$, so that $\sin ^{2} \chi=\left(\hat{\boldsymbol{v}}^{0}-\boldsymbol{x}_{k}^{0}\left(\hat{\boldsymbol{v}}^{0} \cdot \boldsymbol{x}_{k}^{0}\right)\right)^{2}$. The polar angles of $\hat{\boldsymbol{v}}^{0}$ are denoted by $\left(\theta^{\prime}, \varphi^{\prime}\right)$, so that $\cos \theta^{\prime}=x^{0} \cdot \hat{\boldsymbol{v}}^{0}$. We write $\sin ^{2} \chi=\frac{2}{3}\left(1-P_{2}(\cos \chi)\right)$ and use the addition theorem $P_{2}(\cos \chi)=P_{2}(\cos \theta) P_{2}\left(\cos \theta^{\prime}\right)+\cdots$, the omitted terms depend on factors $\mathrm{e}^{\mathrm{i} n \varphi}$ which drop out in the $\varphi$-integration in (5.8). The $\theta$-integration is standard and we find for the angular integral (5.8)

$$
A \sim \frac{2 \pi c \sin ^{2} \theta^{\prime}}{\mathrm{i} \omega n_{0} r}\left(\exp \left(\mathrm{i} \omega n_{0} r / c\right)-\exp \left(-\mathrm{i} \omega n_{0} r / c\right)\right) .
$$

Making use of (5.2), we may write (5.7) as

$$
\left(\hat{\boldsymbol{E}}^{\mathrm{abs}}(\boldsymbol{x}, \omega) \cdot \hat{\boldsymbol{v}}^{0}\right) \hat{\boldsymbol{v}}^{0} \sim \frac{\mathrm{i} \omega q}{8 \pi c^{2}} \frac{1}{r}\left(\mathrm{e}^{\mathrm{i} \omega n_{0} r / c}-\mathrm{e}^{-\mathrm{i} \omega n_{0} r / c}\right) \sin ^{2}\left(\boldsymbol{x}, \hat{\boldsymbol{v}}^{0}\right) \hat{\boldsymbol{v}} .
$$

This is the advanced absorber field generated by the oscillators of the ether as a response to the retarded field (5.1) of the source, more precisely, as a response to the time-symmetric field of the source, which, when added to the advanced absorber field, results in the retarded field (5.1). This retarded field then triggers, self-consistently, the advanced absorber field. For comparison, the velocity component of (5.1) reads as

$$
\left(\hat{\boldsymbol{E}}^{\mathrm{ret}}(\boldsymbol{x}, \omega) \cdot \hat{\boldsymbol{v}}^{0}\right) \hat{\boldsymbol{v}}^{0} \sim \frac{\mathrm{i} \omega q}{4 \pi c^{2}} \frac{1}{r} \mathrm{e}^{\mathrm{i} \omega n_{0} r / c} \sin ^{2}\left(\boldsymbol{x}, \hat{\boldsymbol{v}}^{0}\right) \hat{\boldsymbol{v}}
$$

where we have dropped the infinitesimal damping factor, that is, replaced $n(\omega)$ by $n_{0}(\omega)$. The advanced field $\hat{\boldsymbol{E}}^{\mathrm{adv}}$ of the source is likewise given by $(5.11)$, but with a minus sign in the exponential, $\mathrm{cf}$ (2.14). This suggests the relation

$$
\hat{\boldsymbol{E}}^{\mathrm{abs}}=\frac{1}{2}\left(\hat{\boldsymbol{E}}^{\mathrm{ret}}-\hat{\boldsymbol{E}}^{\mathrm{adv}}\right) .
$$

Clearly, this derivation of (5.12) holds only asymptotically and for the velocity projection, and should be regarded as a first guess, an indication that an absorber field and thus an absorber exists and that (5.12) holds as an identity, also for the vector potential. If so, we may write the retarded field as $\hat{\boldsymbol{E}}^{\mathrm{ret}}=\hat{\boldsymbol{E}}^{\mathrm{sym}}+\hat{\boldsymbol{E}}^{\mathrm{abs}}$, with the initial time-symmetric $\hat{\boldsymbol{E}}^{\mathrm{sym}}:=\frac{1}{2}\left(\hat{\boldsymbol{E}}^{\mathrm{ret}}+\hat{\boldsymbol{E}}^{\mathrm{adv}}\right)$ generated by the Green function (2.10) (with $k(\omega)$ in (4.4)) via (2.7). Here and in the following the superscripts ret, adv and sym refer to the respective fields of the source; the advanced absorber field is denoted by abs, as in (5.10).

The above derivation of the absorber field is approximate and one would like to make sure that there is no causality violation at all, no observable advanced component, not even a 
tiny one, even at short length scales. Though there is no proper retarded Green function for superluminal wave motion, strictly retarded wave propagation is quite easy to derive directly from the field equations (2.2) or (4.2), without explicit reference to the microscopic oscillators. First, we consider $\hat{\varepsilon}(\omega)=\hat{\mu}(\omega)=1$, i.e. the dispersion relation in (2.9) and the wave equation (2.2). Exact retarded and advanced wave solutions have already been obtained in (2.14), $A_{\mu}^{\text {ret/adv }}=2 A_{\mu}^{ \pm}$, Fourier transformed according to (2.12). The field $A_{\mu}^{\text {sym }}=A_{\mu}^{+}+A_{\mu}^{-}$ is generated by the convolution (2.7) of the time-symmetric Green function (2.8) with the current. We now show that the absorber field $A_{\mu}^{\mathrm{abs}}=A_{\mu}^{+}-A_{\mu}^{-}$, evidently a solution of the homogeneous wave equation (2.2), can likewise be obtained by convolutions with the current, the key distribution being

$D^{\mathrm{abs}}(t, \boldsymbol{x}):=\frac{1}{4 \pi^{2} c r} \int_{0}^{\infty} \mathrm{d} \omega \cos (\omega t) \sin (k(\omega) r)=-\Delta_{2}\left(t \rightarrow c t, m \rightarrow m_{\mathrm{t}}\right)$,

with positive $k(\omega)$ as defined in (2.9). This singular solution $\Delta_{2}$ of the homogeneous wave equation (2.2) is calculated in (A.6). The integral in (5.13) can either be defined by an $\varepsilon$-regularizer, as done in $(2.10)$, or, in this case more conveniently, by differentiation of the discontinuous integral $\int_{0}^{\infty} \mathrm{d} \omega k^{-1}(\omega) \cos (\omega t) \cos (k(\omega) r)$, cf [28]. The absorber field is obtained as

$A_{\mu}^{\mathrm{abs}}(x)=\int_{R^{4}} D^{\mathrm{abs}}\left(x-x^{\prime}\right) j_{\mu}^{\mathrm{abs}}\left(x^{\prime}\right) \mathrm{d} x^{\prime}$,

$j_{\mu}^{\mathrm{abs}}(t, \boldsymbol{x}):=\frac{1}{\pi} \int_{-\infty}^{+\infty} j_{\mu}\left(t-t^{\prime}, \boldsymbol{x}\right) P \frac{1}{t^{\prime}} \mathrm{d} t^{\prime}=\frac{\mathrm{i}}{2 \pi} \int_{-\infty}^{\infty} \mathrm{d} \omega \operatorname{sign}(\omega) \hat{j}_{\mu}(\boldsymbol{x}, \omega) \mathrm{e}^{-\mathrm{i} \omega t}$,

where $j^{\mu}$ is of course the current in the wave equation (2.2), and $\hat{j}^{\mu}$ is its Fourier transform according to (2.12). (The $\varepsilon$-regularization of the principal value indicated after (2.11) can be used here.) Equation (5.14) is most easily derived in Fourier space, by means of (2.14). Retarded and advanced fields can be separated in a clear-cut way according to $A_{\mu}^{\text {ret, adv }}=A_{\mu}^{\text {sym }} \pm A_{\mu}^{\text {abs }}$, where $A_{\mu}^{\text {sym }}$ is defined by (2.10) and (2.7), and $A_{\mu}^{\text {abs }}$ by the solution (5.13)-(5.15) of the homogeneous wave equation (2.2).

To comprehend the meaning of (5.13)-(5.15), we symbolically rearrange the integrations in (5.14) and (5.15), so that $A_{\mu}^{\mathrm{abs}}=\int P D^{\mathrm{abs}}\left(x-x^{\prime}\right) j_{\mu}\left(x^{\prime}\right) \mathrm{d} x^{\prime}$, with $P D^{\mathrm{abs}}(t):=$ $\pi^{-1} \int D^{\text {abs }}\left(t-t^{\prime}\right) P t^{\prime-1} \mathrm{~d} t^{\prime}$. This convolution reminds one of the subluminal equation $\frac{1}{2}\left(G^{\text {ret }}-G^{\text {adv }}\right)$ of the homogeneous wave equation (with positive mass-square) antisymmetric in time. $P D^{\text {abs }}$ as defined by the successive integrations (5.15) and (5.14) is a substitute for the exponentially diverging Pauli-Jordan function, $\mathrm{cf}$ (A.1), and the superluminal analogue to the retarded Green function is, symbolically, $G^{\mathrm{sym}}+P D^{\mathrm{abs}}$.

As for retarded and advanced modes in a refractive and absorptive spacetime, we write in Fourier space, by means of (2.14) and still with $\hat{\varepsilon}=\hat{\mu}=1$,

$$
\begin{aligned}
& \hat{A}_{\mu}^{\mathrm{sym}}(\boldsymbol{x}, \omega)=\frac{1}{4 \pi c} \int_{R^{3}} \hat{j}_{\mu}\left(\boldsymbol{x}^{\prime}, \omega\right) \frac{\cos \left(\operatorname{sign}(\omega) k(\omega)\left|\boldsymbol{x}-\boldsymbol{x}^{\prime}\right|\right)}{\left|\boldsymbol{x}-\boldsymbol{x}^{\prime}\right|} \mathrm{d} \boldsymbol{x}^{\prime}, \\
& \hat{A}_{\mu}^{\mathrm{abs}}(\boldsymbol{x}, \omega)=\frac{1}{4 \pi c} \int_{R^{3}} \hat{j}_{\mu}\left(\boldsymbol{x}^{\prime}, \omega\right) \frac{\mathrm{i} \sin \left(\operatorname{sign}(\omega) k(\omega)\left|\boldsymbol{x}-\boldsymbol{x}^{\prime}\right|\right)}{\left|\boldsymbol{x}-\boldsymbol{x}^{\prime}\right|} \mathrm{d} \boldsymbol{x}^{\prime}, \\
& \hat{A}_{\mu}^{\mathrm{ret}, \mathrm{adv}}(\boldsymbol{x}, \omega)=\hat{A}_{\mu}^{\mathrm{sym}} \pm \hat{A}_{\mu}^{\mathrm{abs}} .
\end{aligned}
$$

The Fourier transforms are defined in (2.12), and $\hat{j}_{\mu}=\left(-c^{2} \hat{\rho}, \hat{j}\right), \operatorname{cf}(2.14) . k(\omega)$ is positive as in (2.9). Equations (5.16)-(5.18) remain valid for frequency-dependent complex permeabilities if we replace $\operatorname{sign}(\omega) k(\omega)$ by $\omega n(\omega) / c$, cf (4.4). In the Fourier integral in (5.13), $k(\omega)$ 
is likewise replaced by $\omega n(\omega) / c$. (The real-space expressions in the appendix have to be modified accordingly.) Equations (5.14) and (5.15) remain unaltered. It is easy to check that the wave fields (5.18) with the mentioned replacement satisfy the Maxwell equations (4.2), with field strengths and inductions as defined in (2.3), (4.1) and (4.3).

\section{Conclusion}

We have studied superluminal wave propagation in the ether, a permeable spacetime, and investigated the generation of retarded superluminal modes from subluminal currents. To this end the ether is considered as absorber whose microscopic oscillators carry tachyonic charge and change the advanced components of time-symmetric tachyon fields into retarded ones. Wheeler and Feynman designed the absorber theory for electrodynamics, and they assumed the absorber field to be generated by the collection of charged particles in the universe. At first sight this seems absurd, if one imagines that one-half of the force acting between two nearby charges should stem from the ions of distant galaxies. Clearly, the absorber theory strongly reminds one of the Mach principle, of the attempt to explain the inertial force in Newton's equations as a gravitational reaction of the universe. Both theories have never been satisfactorily incorporated into the contemporary physical world view, based on local interactions and the relativity principle. In fact, one may regard it precisely as the strength of Newton's and Maxwell's equations and their quantum refinements, that it is not necessary to take the universe into account when describing a physical process, a few local interactions will do. Yet, such local descriptions also have their limits, depending on arbitrary input parameters such as particle masses and coupling constants. And so one may ask whether one can understand these constants better, e.g. their dimensionless ratios, if these laws are put into a cosmological context $[21,33,34]$, and the Mach principle as well as the Wheeler-Feynman theory aim in this direction, even though locally one can do without them.

As for relativity theory, a cosmic absorber is conceptually remote from the relativistic interpretation of Lorentz transformations, indigenous to locally geodesic coordinate frames, to the void of Minkowski space. It seems to me more promising to deal with the Mach principle and the absorber theory in an absolute space conception, a permeable ether with an absolute cosmic time provided by the comoving galaxy frame. This is the natural setting for superluminal wave propagation, as the causality of events connected by superluminal signals is then unambiguously defined by the cosmic time order.

For electromagnetic and tachyonic wave propagation alike, the only observable fields are retarded, because all other fields considered in section 5 are acausal, containing advanced components. Nevertheless, a similar mechanism as outlined in the phenomenological derivation of the absorber field can turn time-symmetric modes into completely advanced ones. This is not surprising, as the field equations admit retarded and advanced solutions on an equal footing, cf (5.18). Entropy arguments and the cosmic space expansion have been suggested to break this time symmetry and to justify retarded rather than advanced solutions $[26,35]$. Clearly, the cosmic time evolution and the global 3-space structure should be incorporated, in particular the cosmic time scaling of $N_{0} q_{0}^{2} / m_{0}$ in (5.2); some preliminary studies of the Proca equation in hyperbolic Robertson-Walker cosmologies can be found in [36]. There is also no doubt that statistical averaging is involved when relating the macroscopic permeabilities to the oscillators of the ether. However, it is a little overdone to invoke thermodynamics and space expansion to discard advanced solutions, as causality already takes care of that. The formal possibility of advanced solutions need not be a matter of concern. After all, it is easy to define acausal initial or terminal conditions for Newton's equations, or to define rays which correspond in the geometrical optics limit to advanced wave fields; the formal existence 
of acausal solutions in no way limits the usefulness of causal, retarded wave propagation. Causality is not a physical principle and is in no need of thermodynamic or cosmological explanations, it is just the other way round. However, apparently there are also other views in this regard, and electromagnetic advanced fields have been searched for on the grounds that the absorber field in an expanding spacetime could possibly only incompletely cancel the advanced modes of the initial time-symmetric field [37].

\section{Acknowledgments}

The author acknowledges the support of the Japan Society for the Promotion of Science. The hospitality and stimulating atmosphere of the Centre for Nonlinear Dynamics, Bharathidasan University, Trichy, the Institute of Mathematical Sciences, Madras, and the Tata Institute of Fundamental Research, Bombay, and the Indian Institute of Astrophysics, Bangalore, are likewise gratefully acknowledged. I would like to thank Nandor Balazs and George Contopoulos for extensive discussions on causality.

\section{Appendix. Green functions for negative mass-square}

The inversion problem (2.7) for negative mass-square can readily be solved by analytic continuation, $m \rightarrow \pm \mathrm{i} m, m>0$, of the real-space Green functions for positive mass-square listed in [38]. We use the same notation as in this reference: $D(m)$ for the Pauli-Jordan function, $D^{\text {ret/adv }}(m)$ for retarded and advanced Green functions, and $D^{\text {c/a }}(m)$ for Feynman and Dyson propagators, respectively, and we put $\hbar=c=1$. The analytic continuation of $D(m)$ is

$$
D(\mathrm{i} m)=\frac{1}{2 \pi} \varepsilon(t) \delta\left(r^{2}-t^{2}\right)+\frac{m}{4 \pi} \varepsilon(t) \theta\left(t^{2}-r^{2}\right) \frac{I_{1}\left(m \sqrt{t^{2}-r^{2}}\right)}{\sqrt{t^{2}-r^{2}}},
$$

and $D(\mathrm{i} m)=D(-\mathrm{i} m)$, so that retarded and advanced Green functions admit the continuations $D^{\text {ret }}(\mathrm{i} m)=\theta(t) D(\mathrm{i} m)$ and $D^{\text {adv }}(\mathrm{i} m)=-\theta(-t) D(\mathrm{i} m)$. These distributions solve

$$
\left(\square+m^{2}\right) D(\mathrm{i} m)=0, \quad\left(\square+m^{2}\right) D^{\mathrm{ret} / \mathrm{adv}}(\mathrm{i} m)=-\delta(t) \delta(\boldsymbol{x}),
$$

with $\square=-\partial^{2} / \partial t^{2}+\Delta$. The continuation of the time-symmetric Green function $D^{\text {sym }}=$ $\frac{1}{2}\left(D^{\text {ret }}+D^{\mathrm{adv}}\right)$ is accordingly

$$
D^{\mathrm{sym}}(\mathrm{i} m)=\frac{1}{4 \pi} \delta\left(r^{2}-t^{2}\right)+\frac{m}{8 \pi} \frac{\theta\left(t^{2}-r^{2}\right)}{\sqrt{t^{2}-r^{2}}} I_{1}\left(m \sqrt{t^{2}-r^{2}}\right),
$$

and we find the identities $D^{\text {ret/adv }}(\mathrm{i} m)=2 \theta( \pm t) D^{\text {sym }}(\mathrm{i} m)$ and $D(\mathrm{i} m)=2 \varepsilon(\tau) D^{\text {sym }}(\mathrm{i} m)$. The mass $m$ on the right-hand side of (A.1) and (A.3) is the tachyon mass; the natural units are restored by substituting $m \rightarrow m_{\mathrm{t}} c / \hbar$ and $t \rightarrow c t$ into (A.1), (A.3) and (A.6) below. The continuation to imaginary mass was effected by $J_{1}( \pm \mathrm{i} x)= \pm \mathrm{i} I_{1}(x)$ and

$N_{1}( \pm \mathrm{i} x)=-I_{1}(x) \pm \frac{2}{\pi} \mathrm{i} K_{1}(x), \quad K_{1}( \pm \mathrm{i} x)=-\frac{\pi}{2}\left(J_{1}(x) \mp \mathrm{i} N_{1}(x)\right)$,

where $x>0$, cf [28]. The analytic continuations of Feynman and Dyson propagators read

$D^{\mathrm{c}}( \pm \mathrm{i} m)=D^{\mathrm{sym}}(\mathrm{i} m)+\frac{1}{2}\left( \pm \Delta_{1}-\mathrm{i} \Delta_{2}\right)$

$D^{\mathrm{a}}( \pm \mathrm{i} m)=D^{\mathrm{sym}}(\mathrm{i} m)-\frac{1}{2}\left( \pm \Delta_{1}-\mathrm{i} \Delta_{2}\right)$, 
$\Delta_{1}:=\frac{m}{4 \pi} \theta\left(t^{2}-r^{2}\right) \frac{I_{1}\left(m \sqrt{t^{2}-r^{2}}\right)}{\sqrt{t^{2}-r^{2}}}+\frac{m}{4 \pi} \theta\left(r^{2}-t^{2}\right) \frac{J_{1}\left(m \sqrt{r^{2}-t^{2}}\right)}{\sqrt{r^{2}-t^{2}}}$,

$\Delta_{2}:=\frac{m}{2 \pi^{2}} \theta\left(t^{2}-r^{2}\right) \frac{K_{1}\left(m \sqrt{t^{2}-r^{2}}\right)}{\sqrt{t^{2}-r^{2}}}+\frac{m}{4 \pi} \theta\left(r^{2}-t^{2}\right) \frac{N_{1}\left(m \sqrt{r^{2}-t^{2}}\right)}{\sqrt{r^{2}-t^{2}}}$.

Clearly, $D^{\mathrm{a}}( \pm \mathrm{i} m)=\overline{D^{\mathrm{c}}(\mp \mathrm{i} m)}$, and $\left(\square+m^{2}\right) \Delta_{1,2}=0$, and

$\Delta_{1}=2 D^{\mathrm{sym}}(\mathrm{i} m)-D^{\mathrm{c}}(-\mathrm{i} m)-D^{\mathrm{a}}(\mathrm{i} m), \quad \Delta_{2}=\mathrm{i}\left(D^{c}(-\mathrm{i} m)-D^{a}(\mathrm{i} m)\right)$.

The complete solution of $\left(\square+m^{2}\right) G=-\delta$ is

$$
G=\alpha D^{\mathrm{ret}}(\mathrm{i} m)+(1-\alpha) D^{\mathrm{adv}}(\mathrm{i} m)+c_{1} \Delta_{1}+c_{2} \Delta_{2},
$$

with arbitrary complex constants $\alpha$ and $c_{1,2}$. The only solution not supported inside the lightcone is the real part of $D^{\mathrm{c}}(-\mathrm{i} m)$, i.e. $\alpha=-c_{1}=\frac{1}{2}, c_{2}=0$, used in (2.8):

$$
G^{\mathrm{sym}}=D^{\mathrm{sym}}(\mathrm{i} m)-\frac{1}{2} \Delta_{1}=\frac{1}{2}\left(D^{\mathrm{c}}(-\mathrm{i} m)+D^{\mathrm{a}}(\mathrm{i} m)\right) .
$$

Clearly, $G^{\text {sym }}$, rather than $D^{\text {sym }}(\mathrm{i} m)$, is the tachyonic analogue to the subluminal $D^{\text {sym }}(m)$ supported on the interior lightcone and symmetric with respect to time inversions. In particular, there is no tachyonic analogue to $D^{\text {ret }}(m)$ and $D^{\text {adv }}(m)$, i.e. a propagator supported on the exterior of the lightcone and vanishing for $t<0$ or $t>0$, respectively. This is no surprise, as $D^{\text {ret }}(m)$ and $D^{\text {adv }}(m)$ are defined on two disconnected components, whereas the exterior of the cone is connected, and Lorentz transformations do not preserve the time order of events with spacelike connection. The only singularity of $G^{\mathrm{sym}}$ stems from the $\delta$-function; $J_{1}(z) / z$ is regular and decays at infinity. Singular functions not containing the diverging $I_{1}(z)$ are linear combinations of $D^{\mathrm{c}}(-\mathrm{i} m)$ and $D^{\mathrm{a}}(\mathrm{i} m)$. The only homogeneous solution not exponentially diverging is $\Delta_{2}$ in (A.6), supported in and outside the cone, which is used in section 5, together with $G^{\text {sym }}$, to generate retarded wave fields, cf (5.13).

Remark. The inverting kernel of the Proca equation (2.1) is $G_{\alpha \beta}(t, x):=\left(\eta_{\alpha \beta}+\right.$ $\left.m_{\mathrm{t}}^{-2} \partial_{\alpha} \partial_{\beta}\right) G(t, \boldsymbol{x})$, to be used instead of the scalar Green function in the case of a nonconserved current. For instance, the current is not conserved if the tachyonic charge depends on cosmic time. Clearly, $G_{\alpha, \beta}^{\beta}=-c^{-1} m_{\mathrm{t}}^{-2} \delta_{, \alpha}$, cf (2.7). We consider the bivector $G_{\alpha \alpha^{\prime}}\left(x, x^{\prime}\right):=\left(\eta_{\alpha \alpha^{\prime}}-m_{t}^{-2} \partial_{\alpha} \partial_{\alpha^{\prime}}\right) G\left(x-x^{\prime}\right)$, and define $F_{\alpha \alpha^{\prime} \beta}^{G}\left(x, x^{\prime}\right):=G_{\beta \alpha^{\prime}, \alpha}-G_{\alpha \alpha^{\prime}, \beta}$, which is a second-rank skew tensor with respect to $x$, and a vector with respect to $x^{\prime}$. (Primed indices refer to the primed variable.) We find

$$
F_{\alpha \alpha^{\prime}, \beta}^{G \beta}-m_{\mathrm{t}}^{2} G_{\alpha \alpha^{\prime}}=c^{-1} \delta\left(x-x^{\prime}\right) \eta_{\alpha \alpha^{\prime}},
$$

so that $A_{\alpha}=\int G_{\alpha \alpha^{\prime}}\left(x-x^{\prime}\right) j^{\alpha^{\prime}}\left(x^{\prime}\right) \mathrm{d} x^{\prime}$ solves the field equations (2.1) according to

$$
F_{\alpha}{ }^{\beta}, \beta-m_{\mathrm{t}}^{2} A_{\alpha}=\int\left(F_{\alpha \alpha^{\prime}, \beta}^{G, \beta}-m_{\mathrm{t}}^{2} G_{\alpha \alpha^{\prime}}\right) j^{\alpha^{\prime}}\left(x^{\prime}\right) \mathrm{d} x^{\prime}=c^{-1} j_{\alpha} .
$$

Replacing $G j_{\alpha}$ by $G_{\alpha \alpha^{\prime}} j^{\alpha^{\prime}}$ in (2.7), we obtain $\left(\square+m_{\mathrm{t}}^{2}\right) A_{\alpha}=-c^{-1}\left(j_{\alpha}+m_{\mathrm{t}}^{-2} j^{\beta}, \beta, \alpha\right)$. The scalar Green function inverts the four Klein-Gordon equations in (2.2). If $j^{\beta}, \beta=0$, the vector potential defined in (2.7) also satisfies the Lorentz condition (easy to see via Gauss theorem), so that we need not use the cumbersome bivector $G_{\alpha \alpha^{\prime}}$ in the calculations of this paper. 


\section{References}

[1] Sommerfeld A 1904 Proc. Konink. Akad. Wet. (Sec. Sci.) 7346

[2] Brillouin L 1960 Wave Propagation and Group Velocity (New York: Academic)

[3] Tanaka S 1960 Prog. Theor. Phys. 24171

[4] Terletsky Ya P 1961 Sov. Phys.-Dokl. 5782

[5] Feinberg G 1967 Phys. Rev. 1591089

[6] Parker L 1969 Phys. Rev. 1882287

[7] Feinberg G 1970 Sci. Am. 22269

[8] Newton R 1970 Science 1671569

[9] Pirani F A E 1970 Phys. Rev. D 13224

[10] Fuller R W and Wheeler J A 1962 Phys. Rev. 128919

[11] Feinberg G 1978 Phys. Rev. D 171651

[12] Anderson R, Vetharaniam I and Stedman G E 1998 Phys. Rep. 29593

[13] Tomaschitz R 1999 Int. J. Mod. Phys. A 145137

[14] Tomaschitz R 2000 Eur. Phys. J. B 17523

[15] Goldhaber A S and Nieto M M 1971 Rev. Mod. Phys. 43277

[16] Tomaschitz R 2000 Celest. Mech. Dyn. Astron. 77107

[17] Whittaker E 1951 A History of the Theories of Aether and Electricity (London: Nelson)

[18] Tomaschitz R 1998 Chaos Solitons Fractals 91199

[19] Tomaschitz R 1998 Int. J. Theor. Phys. 371121

[20] Tomaschitz R 1998 Astrophys. Space Sci. 259255

[21] Tomaschitz R 2000 Astrophys. Space Sci. 271181

[22] Volkov A M and Kiselev V A 1970 Sov. Phys.-JETP 30733

[23] Mashhoon B 1973 Phys. Rev. D 84297

[24] Sandage A 1988 Ann. Rev. Astron. Astrophys. 26561

[25] Tomaschitz R 2001 Physica A 293225

[26] Wheeler J A and Feynman R P 1945 Rev. Mod. Phys. 17157

[27] Proca A 1936 J. Physique 7347

[28] Watson G N 1996 A Treatise on the Theory of Bessel Functions (Cambridge: Cambridge University Press)

[29] Landau L D and Lifshitz E M 1984 Electrodynamics of Continuous Media (Oxford: Pergamon)

[30] Jackson J D 1999 Classical Electrodynamics 3rd edn (New York: Wiley)

[31] Dirac P A M 1937 Proc. R. Soc. A 167148

[32] Plass G N 1961 Rev. Mod. Phys. 3337

[33] Dirac P A M 1973 The Physicist's Conception of Nature ed J Mehra (Dordrecht: Reidel)

[34] Dyson F J 1972 Aspects of Quantum Theory ed A Salam and E P Wigner (Cambridge: Cambridge University Press)

[35] Davies P C W 1972 J. Phys. A: Math. Gen. 51722

[36] Tomaschitz R 2001 J. Math. Phys. 42 (12)

[37] Partridge R B 1973 Nature 244263

[38] Bogoliubov N N and Shirkov D V 1980 Introduction to the Theory of Quantized Fields (New York: Wiley) 